\title{
Aggregation under local reinforcement: From lattice to continuum
}

\author{
Dirk Horstmann ${ }^{1}$, Kevin J. Painter ${ }^{2}$ and Hans G. Othmer ${ }^{3}$ \\ ${ }^{1}$ Mathematisches Institut der Universität zu Köln, \\ Weyertal 86-90, D-50923 Köln, Germany, (dhorst@mi.uni-koeln.de). \\ 2 Department of Mathematics, Heriot-Watt University, \\ Riccarton, Edinburgh EH14 4AS, Scotland, (painter@ma.hw.ac.uk). \\ ${ }^{3}$ School of Mathematics, University of Minnesota, \\ Vincent Hall 207a, 206 Church St. S.E., Minneapolis, MN 55455 USA, \\ (othmer@math. umn.edu).
}

(Received 7 October 2002)

\begin{abstract}
Movement of biological organisms is frequently initiated in response to a diffusible or otherwise transported signal, and in its simplest form this movement can be described by a diffusion equation with an advection term. This form was first derived by Patlak [24], but since then it has been re-derived in more generality by many others. However, in some cases the signal is localized in space, as for instance, when organisms deposit a non-diffusible signal that modifies the properties of the local environment. In these systems, one example of which is the myxobacteria, the question arises as to whether aggregation is possible under suitable hypotheses on the transition rules and the production of a control species that modulates the transition rates. It is shown in [21] that continuum limits of reinforced random walks show aggregation and even blow-up, but the connection between solutions of the continuum equations and the lattice walk was not studied. Here we show for certain simplified versions of the model studied earlier that the stable solutions of the lattice walk "converge" to the stable solutions of the corresponding partial differential equation. Thus the passage to the continuum limit seems to be justified in these cases.
\end{abstract}

\section{Introduction}

Living systems sense and respond to their environment, and frequently this involves movement in response to an external stimulus. Such behavior is generally referred to as a taxis, and can be characterized as positive or negative, depending on whether the external stimulus triggers movement towards or away from the source. Many stimuli have been shown to elicit a taxis response, such as chemicals (chemotaxis), oxygen (aerotaxis), and electric fields (galvanotaxis). The mecha- 
nisms leading to a taxis response involve sensing the external signal, transducing it into an internal signal, and modifying movement appropriately. While many instances involve detection and response without alteration of the signal field, often the signal itself is modified as part of the response, thus resulting in a coupling between the local density of individuals and the intensity of the signal. A classical example of the latter occurs in cellular slime molds such as Dictyostelium discoideum which, upon detection of extracellular cyclic adenosine monophosphate (cAMP), both secrete cAMP and respond chemotactically by moving toward the source of cAMP. This process of signal relay to more distant cells and chemotactic movement produces aggregation in large fields of initially-dispersed cells [20].

Frequently the external signal is transported by diffusion, convection, or other means, but in other instances the signal is strictly local, with little or no transport. Examples include the myxobacteria, which produce a non-diffusible "slime" over which their cohorts can move more readily, and ants, which follow trails left by predecessors $[10,5]$. The myxobacteria are ubiquitous soil bacteria which glide on suitable surfaces or at air-water interfaces. Under starvation conditions they demonstrate an increased tendency to glide closer to one another, eventually aggregating to form what are called fruiting bodies. Inside the fruiting bodies they survive as dormant myxospores. During gliding the myxobacteria produce slime trails on which they prefer to glide, and when they encounter a slime trail they typically alter their direction so as to glide on it. Once on the slime trail, the gliding velocity increases. The mechanisms by which myxobacteria propel themselves on the substrate and aggregate are still not understood, and thus theoretical analyses provide a framework for exploring different mechanisms. Here we analyze a class of space-jump processes [19] that may describe their aggregation; elsewhere velocity jump processes have been used for this purpose [16].

It is known that in the classical chemotaxis problem individuals can aggregate in space in response to a diffusible signal produced by the individuals, and in [21] it was shown numerically that aggregation can occur even in the absence of signal transport. The aggregation demonstrated in [21] for an immobile signal is based on the continuum limit of a lattice walk in which the walker modifies the local transition probabilities. Since the aggregation solutions can be sharply concentrated, the a priori bounds on higher derivatives needed to justify the continuum limit may not hold, and the question arises as to what the relationship is between solutions of the lattice walk and those of the partial differential that arises in the continuum limit. We investigate this question in the present paper. Some preliminary results on this question are given in [22]. 


\section{The dynamics of movement}

We begin with a master equation for a continuous-time, discrete-space random walk. In this paper we consider only random walks in one space dimension, but the analysis extends to higher dimensions as well. Whether the localization results also carry over remains to be determined rigorously, but numerical results strongly suggest that the conclusions do not depend on the dimension of the underlying space. As in [21], we consider a lattice walk in which the transition rates depend on the density of a control species that modulates the transition rates. We restrict attention to one-step jumps, although it is easy to generalize this (cf. [4]). However, usually one does not obtain diffusion equations in the continuum limit.

Suppose that the conditional probability $p_{i}(t)$ that a walker is at $n \in \mathbb{Z}$ at time $t$, conditioned on the fact that it begins at $n=0$ at $t=0$, evolves according to the continuous time master equation

$$
\frac{\partial p_{i}}{\partial t}=\hat{\mathcal{T}}_{i-1}^{+}(W) p_{i-1}+\hat{\mathcal{T}}_{i+1}^{-}(W) p_{i+1}-\left(\hat{\mathcal{T}}_{i}^{+}(W)+\hat{\mathcal{T}}_{i}^{-}(W)\right) p_{i} .
$$

Here $\hat{\mathcal{T}}_{i}^{ \pm}(\cdot)$ are the transition probabilities per unit time for a one-step jump to $n \pm 1$, and $\left(\hat{\mathcal{T}}_{i}^{+}(W)+\hat{\mathcal{T}}_{i}^{-}(W)\right)^{-1}$ is the mean waiting time at the $n^{\text {th }}$ site. We assume throughout that these are nonnegative and suitably smooth functions of their arguments. The vector $W$ is given by

$$
W=\left(\cdots, w_{-i-1 / 2}, w_{-i}, w_{-i+1 / 2}, \cdots, w_{o}, w_{1 / 2}, \cdots\right) .
$$

For generality the density of the control species $w$ is defined on the embedded lattice of half the step size. As (2.1) is written, the jump probabilities may depend on the entire state and on the entire distribution of the control species. Since there is no explicit dependence on the previous state the process may appear to be Markovian, but if the evolution of $w_{i}$ depends on $p_{i}$ then there is an implicit history dependence, and thus the jump process by itself is not Markovian. However, the composite process for the evolution $(p, w)$ is a Markov process.

There are three distinct types of models that are considered in [21], which differ in the dependence of the transition rates on $w,:$ (i) strictly local models, (ii) barrier models, and (iii) gradient models. Here we focus on the first of these. We assume that the transition rates $\hat{\mathcal{T}}_{i}^{ \pm}$depend only on $w_{i}$ and we write $\hat{\mathcal{T}}_{i}^{ \pm}=\hat{\mathcal{T}}\left(w_{i}\right)$. That is, we assume that the mean waiting time at site $n$ depends only on the density of the control species at that site. This describes a myopic walker who bases his decisions entirely on local information, and in this scheme (2.1) becomes

$$
\frac{\partial p_{i}}{\partial t}=\hat{\mathcal{T}}^{+}\left(w_{i-1}\right) p_{i-1}+\hat{\mathcal{T}}^{-}\left(w_{i+1}\right) p_{i+1}-\left(\hat{\mathcal{T}}^{+}\left(w_{i}\right)+\hat{\mathcal{T}}^{-}\left(w_{i}\right)\right) p_{i}
$$

Since the transition probabilities are independent of the lattice site and depend only on the local densities, there is no source of spatial bias in the walk and we 
must assume that

$$
\hat{\mathcal{T}}^{+}=\hat{\mathcal{T}}^{-} \equiv \hat{\mathcal{T}}
$$

Then (2.3) becomes

$$
\frac{\partial p_{i}}{\partial t}=\hat{\mathcal{T}}\left(w_{i-1}\right) p_{i-1}+\hat{\mathcal{T}}\left(w_{i+1}\right) p_{i+1}-2 \hat{\mathcal{T}}\left(w_{i}\right) p_{i} .
$$

We set $x=n h$, then reinterpret it as a continuous variable, and extend the definition of $p_{i}$ accordingly. Expansion of the right-hand side as a function of $x$ up to second order in $\mathrm{h}$ gives

$$
\frac{\partial p}{\partial t}=h^{2} \frac{\partial^{2}}{\partial x^{2}}(\hat{\mathcal{T}}(w) p)+\mathcal{O}\left(h^{4}\right) .
$$

We suppose that there is a scaling of the transition rates such that $\hat{\mathcal{T}}(w)=$ $\lambda \mathcal{T}(w)$ and such that

$$
\lim _{\substack{h \rightarrow 0 \\ \lambda \rightarrow \infty}} \lambda h^{2}=\text { constant } \equiv D
$$

exists. Then the diffusion limit of $(2.4)$ is

$$
\frac{\partial p}{\partial t}=D \frac{\partial^{2}}{\partial x^{2}}(\mathcal{T}(w) p)
$$

We suppose that the evolution of the signal or control substance $w$ is given by

$$
\frac{d w}{d t}=p^{2}-\mu w
$$

i.e., we suppose that production of $w$ is proportional to the local density of $p^{2}$. As was shown in [21], linear dependence on the density does not lead to aggregation of the walkers. If we further assume that the production of the signal occurs on a much faster time scale than movement, then the first term in the solution of the resulting singularly-perturbed version of (2.7) is $w=\mu / p^{2}$, and we obtain the master equation

$$
\frac{\partial p}{\partial t}=D \frac{\partial^{2}}{\partial x^{2}}(\mathcal{T}(p) p) .
$$

for a new function $\mathcal{T}$. We impose no-flux boundary conditions to guarantee the conservation of the total probability density or population density, since there are no birth-death or immigration processes in the model. Throughout this paper we will refer to this problem as the singular model. Our goal is to compare the behavior of solutions of the continuum problems, both for the nonsingular problem (2.6) \& (2.7) and the singular problem (2.8), with solutions of the corresponding discretized problems, in order to determine the validity of the continuum equations. Some results on this problem are given in [22] and in this paper we focus mainly 
on the continuous aspects of this comparison. The numerical aspects are studied in more detail in [23]

\section{Analysis of the singular problem}

We begin with the singular problem, which is equation (2.8) with Neumann boundary conditions and suitable initial conditions.

$$
\left.\begin{array}{rlrl}
p_{t} & =(\mathcal{T}(p) p)_{x x}, & & \text { in } Q_{T}:=\Omega \times(0, T) \\
(\mathcal{T}(p) p)_{x} & =0, & & \text { on } \Gamma_{T}:=\partial \Omega \times(0, T) \\
p(x, 0) & =p_{0}(x), & & \text { in } \Omega,
\end{array}\right\}
$$

Here $\Omega$ denotes a bounded interval in $I \subset \mathbb{R}$, which after translation and rescaling of $x$, could be set to $[0,1]$. The no-flux boundary condition implies that

$$
\int_{\Omega} p(x, t) d x=\int_{\Omega} p_{0}(x) d x \quad \forall t \geq 0 .
$$

We suppose that $\mathcal{T}: \mathbb{R} \rightarrow \mathbb{R}$ in $C^{1}(0, \infty)$ is such that $\mathcal{T}(p) p \geq 0$ for all $p \in \mathbb{R}^{+}$, $(\mathcal{T}(p) p)^{\prime} \leq$ const, and $(\mathcal{T}(p) p)^{\prime}$ changes sign exactly once on $[0, \infty)$. Two examples that satisfy these conditions are $\mathcal{T}(p) p=p /\left(K+p^{2}\right)$ and $\mathcal{T}(p) p=p e^{-p}$. Such $\mathcal{T}(p)$ in (3.1) can lead to a backward parabolic equation for suitable initial data.

\subsection{The dependence of well-posedness on the initial data}

Because (3.1) may be a forward-backward problem, we must determine whether the problem is well-posed. The following shows that it is well-posed for sufficiently small initial data, and that for these initial data the solution exists globally in time.

Theorem 3.1 Suppose that $(\mathcal{T}(p) p)^{\prime}=0$ at $p=s_{0}$ and that $(\mathcal{T}(p) p)^{\prime}>0$ for $p \in\left[0, s_{0}\right)$ and $(\mathcal{T}(p) p)^{\prime}<0$ for $p>s_{0}$. Then there exists a nonnegative classical solution in $C^{1,2}\left(Q_{T}\right)$ of problem (3.1) for all $T>0$, provided $p_{0}(x) \in C^{2, \beta}(\Omega)(\beta \in$ $(0,1)), 0 \leq p_{0}(x)<s_{0}$ for all $x \in \bar{\Omega}$ and satisfies the compatibility condition $\left(\mathcal{T}\left(p_{0}\right) p_{0}\right)_{x}=0$ on $\partial \Omega .^{1}$

1 The notation for the various Banach spaces used throughout the paper is given in the Appendix A. 
Proof: First we rewrite (3.1) as

$$
\left.\begin{array}{rlrl}
p_{t} & =\left(\left(\mathcal{T}(p)+p \mathcal{T}^{\prime}(p)\right)_{x} p_{x}\right) & \\
& =\left(b(p) p_{x}\right)_{x}, & & \text { in } Q_{T} \\
b(p) p_{x} & =0, & & \text { on } \Gamma_{T} \\
p(x, 0) & =p_{0}(x), & & \text { in } \Omega .
\end{array}\right\}
$$

Since $\Omega$ is bounded there exists a constant $s_{1}$ such that $\left|p_{0}(x)\right| \leq s_{1}<s_{0}$ in $\bar{\Omega}$. Thus we can consider the modified problem

$$
\left.\begin{array}{rlrl}
p_{t} & =\left(a(p) p_{x}\right)_{x}, & & \text { in } Q_{T} \\
a(p) p_{x} & =0, & & \text { on } \Gamma_{T} \\
p(x, 0) & =p_{0}(x), & & \text { in } \Omega .
\end{array}\right\}
$$

where $a(p)$ is defined as $b(p)$ for $p \leq s_{1}$, and for $p>s_{1}$ as a $C^{1}$-continuation $b_{1}(p)$ of $b(p)$ satisfying the following conditions: $b_{1}(p) \geq c>0, p>s_{1}, b_{1}^{\prime}(p) \leq 0, p>s_{1}$. Problem (3.4) is uniformly parabolic and according to [15, Chapter V, Theorem 7.4] there exists a classical solution $p \in C^{1+\frac{\beta}{2}, 2+\beta}\left(Q_{T}\right)$ of (3.4) for every $T>0$. By a standard argument (define $u=\mathcal{T}(p) p$ and redefine the time), the solution is nonnegative for nonnegative initial data.

Furthermore we see that this classical solution of the modified problem satisfies the following $L^{r}$-estimates:

$$
\begin{aligned}
\frac{d}{d t} \int_{\Omega}|p|^{r} & =r \int_{\Omega} p_{t} p|p|^{r-2} d x=r \int_{\Omega} p|p|^{r-2}\left(a(p) p_{x}\right)_{x} d x \\
& =-r(r-1) \int_{\Omega} a(p)|p|^{r-2}\left|p_{x}\right|^{2} d x \leq-c r(r-1) \int_{\Omega}|p|^{r-2}\left|p_{x}\right|^{2} d x \leq 0 .
\end{aligned}
$$

It follows from these inequalities that the $L^{r}$-norm of the modified problem can be bounded for every $r \in[1, \infty)$ in terms of the corresponding $L^{r}$-norm of the initial data by:

$$
\left(\int_{\Omega}|p(x, t)|^{r} d x\right)^{\frac{1}{r}} \leq\left(\int_{\Omega}\left|p_{0}(x)\right|^{r} d x\right)^{\frac{1}{r}} \forall r \in[2, \infty) .
$$

Thus, as $r \rightarrow \infty$, we see that $\|p(x, t)\|_{L^{\infty}(\Omega)} \leq\left\|p_{0}(x)\right\|_{L^{\infty}(\Omega)} \leq s_{1} \forall t>0$. Thus the $L^{\infty}$-norm of the modified problem remains uniformly bounded by the $L^{\infty}$-norm of the initial data for all times, and consequently, the solution $p$ of the modified problem is also a classical solution of the original problem.

These conditions on the initial data lead us to the following definition.

Definition 3.2 We say that the initial data $p_{0}(x)$ for problem (3.1) is subcritical 
if $\left(\mathcal{T}\left(p_{0}\right) p_{0}\right)^{\prime}(x)>0$ in $\Omega$. We call $p_{0}(x)$ supercritical if there exists an $\Omega_{0} \subset \Omega$ such that $\left(\mathcal{T}\left(p_{0}\right) p_{0}\right)^{\prime}(x)<0$ for all $x \in \Omega_{0}$.

Thus there exists a unique classical solution of (3.1) for initial data that is sufficiently smooth and subcritical. Furthermore, an energy argument shows that the solution must converge to the homogeneous distribution in this case. To prove this, let $\Phi(p)=\int T(p) p d p$ be an indefinite integral of $T(p) p$, and consider the energy functional

$$
\mathcal{E}_{c}(t):=\int_{\Omega} \Phi(p(x)) d x
$$

Along trajectories of (3.1)

$$
\frac{d}{d t} \mathcal{E}_{c}(t)=-\int_{\Omega}\left|(\mathcal{T}(p(x, t)) p(x, t))_{x}\right|^{2} d x \leq 0,
$$

and vanishes only when $(\mathcal{T}(p(x, t)) p(x, t))_{x}$ vanishes, and therefore $\mathcal{E}_{c}$ is strictly non-increasing along solutions of (3.1). Since $p(x, t)$ exists globally in time, and is a classical solution for subcritical initial data, we conclude that

$$
\int_{\Omega}\left|(\mathcal{T}(p(x, t)) p(x, t))_{x}\right|^{2} d x \rightarrow 0, \text { as } t \rightarrow \infty .
$$

Therefore $\mathcal{T}(p(x, t)) p(x, t) \rightarrow$ const as $t \rightarrow \infty$, which implies that $p(x, t) \rightarrow$ const, as $t \rightarrow \infty$. The mass conservation condition implies that this constant is given by

$$
M:=\frac{1}{|\Omega|} \int_{\Omega} p_{0}(x) d x .
$$

Thus the case of subcritical initial data is completely understood.

However we do not have a proof for the local existence of a solution of (3.1) for arbitrary supercritical initial data, and numerical computations indicate why this is a difficult issue to resolve. In that figure we show numerically-computed solutions for various sub- and supercritical initial data. As we can see from Figure 1 (c) and (d), the asymptotic behavior of solutions for supercritical initial data can be either numerical blow-up or convergence to the homogeneous solution, and of course the latter implies the global existence of the solution.

The results for a related problem introduced in [25] can be used to show that a solution of (3.1) can only exist, even locally in time, if the initial data is infinitely differentiable. To see this, let

$$
u(x, t):=\int_{0}^{x} p(s, t) d s .
$$



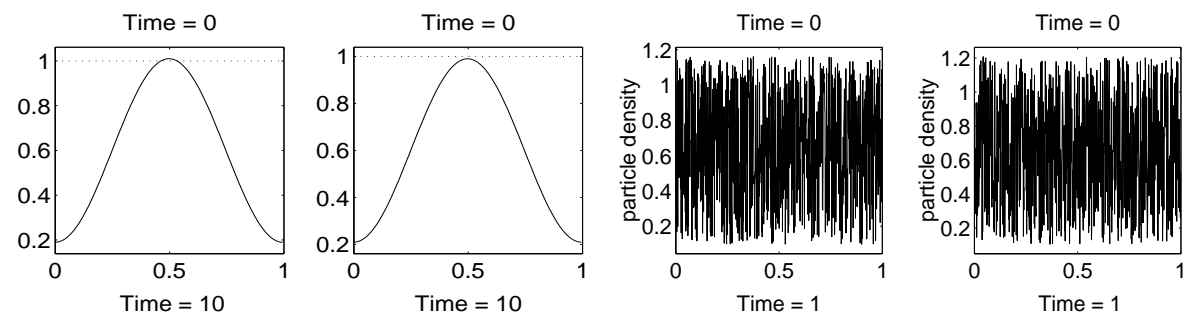

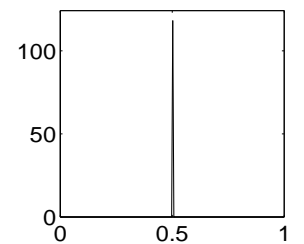

(a)

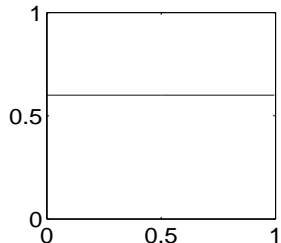

(b)

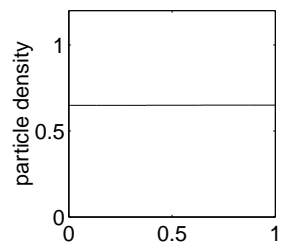

(c)

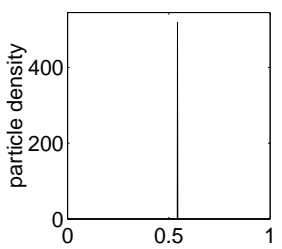

(d)

FIgURE 1. The time evolution of the singular model for $\mathcal{T}(p)=1 /\left(1+p^{2}\right)$ and different initial distributions. For this $\mathcal{T}$ the critical value is $p=1$. (a) $p(x, 0)=0.6-0.4 \cos (2 \pi x)$. Initial particle density is supercritical, and a single high density peak forms. (b) $p(x, 0)=$ $0.6-0.39 \cos (2 \pi x)$. Initial conditions are subcritical, and the homogeneous solution results. (c) and (d) While $\max p(x, 0)<1.0$ is a sufficient criterion for the homogeneous solution to develop, $\max p(x, 0)>1.0$ is not sufficient for the heterogeneous solution to form. Two different sets of initial data for the same initial mass, $M$, can lead to either homogeneous or heterogeneous solutions.

and thereby transform (3.1) into the following Dirichlet problem

$$
\begin{aligned}
u_{t} & =\left(\mathcal{T}\left(u_{x}\right) u_{x}\right)_{x}, \text { in }(0,1) \times(0, T) \\
u(0, t) & =0, u(1, t)=M=\int_{0}^{1} p_{0}(x) d x, u(x, 0)=\int_{0}^{x} p_{0}(s) d s .
\end{aligned}
$$

Equation (3.11) with homogeneous Neumann boundary data and $\mathcal{T}\left(u_{x}\right)$ as described above is used in image enhancement processes and is known as the PeronaMalik [25] equation. In [13] it is shown that a weak solution of this equation satisfying

$$
\int_{0}^{T} \int_{\Omega}\left(\varphi_{t} u-\varphi_{x} \mathcal{T}\left(u_{x}\right) u_{x}\right) d x d t=0
$$

for all $\varphi \in C_{0}^{1}\left(Q_{T}\right)$ can only exist locally if the initial data is infinitely differentiable. Furthermore, in [12] it is shown that if a solution of the Perona-Malik equation with homogeneous Neumann boundary data exists for initial data with $\left|u_{x}(x, 0)\right|>1$ in some region of $\Omega$, then the gradient of this solution blows up in finite time. Figure 1 (c) and (d) suggest that finding criteria for global existence vs. blowup is more subtle in the present problem. 


\subsection{Least energy solutions and connections with the discrete steady state analysis}

For the remainder of the paper we use the particular form $\mathcal{T}(p)=1 /\left(1+p^{2}\right)$ for the transition rate function. According to (3.7), we know that the energy decreases whenever the solution of (3.1) exists, and thus we begin by studying the minimizing problem

$$
\inf _{p \in \mathcal{D}} \mathcal{E}_{c}(p)=\inf _{p \in \mathcal{D}} \int_{\Omega} \frac{1}{2} \log \left(1+p^{2}\right) d x,
$$

where

$$
\mathcal{D}:=\left\{p \in L^{1}(\Omega)\left|p \geq 0, \int_{\Omega} p(x) d x=M\right| \Omega \mid\right\} .
$$

We know from the calculus of variations that either convexity or coercivity and lower semicontinuity of the functional $\mathcal{E}_{c}$ is sufficient to ensure the existence of a minimizer, but $\mathcal{E}_{c}$ is neither coercive nor convex. Consequently we do not know whether or not a minimizer exists, and if one does, whether it is unique (see for example [9, Chapter 8, pp. 431 - 490] on the theory of when a minimization problem achieves its infimum).

Remark 3.3 We see that for $\Omega=(0,1)$ the sequence

$$
\left\{p_{\mu}(x)\right\}_{\mu \geq 0}:=\left\{\frac{1}{x+\mu}\right\}_{\mu \geq 0}
$$

satisfies $\left\|p_{\mu}\right\|_{L^{1}(\Omega)} \rightarrow \infty$ as $\mu \rightarrow 0$ and

$$
\int_{0}^{1} \log \left(1+\left(p_{\mu}(x)\right)^{2}\right) d x<\infty \text { for all } \mu .
$$

This shows the non-coercivity of the functional $\mathcal{E}_{c}$ on $L^{1}(\Omega)$. Furthermore we see that the sequence $\left\{q_{\mu}(x)\right\}_{\mu \geq 0}$ with

$$
q_{\mu}(x):=M \frac{p_{\mu}(x)}{\int_{0}^{1} p_{\mu}(x) d x}
$$

satisfies the mass constraint and is a minimizing sequence of the functional in our considered set since

$$
\int_{0}^{1} \log \left(1+\left(q_{\mu}(x)\right)^{2}\right) d x \rightarrow 0 \text { as } \mu \rightarrow 0 \text { and } \int_{0}^{1} q_{\mu}(x) d x=M \text { for all } \mu .
$$

However one easily can also construct a minimizing sequence with maximum in the other corner of the interval. Therefore one should expect the non uniqueness of a minimizer. 
Thus we have to look to previous results for the discretized version of (3.1) for guidance. The steady state analysis for the discrete formulation

$$
\frac{d P}{d t}=\Delta_{d}(\mathcal{T}(P) P)
$$

of (3.1) done in [22] shows that stationary solutions $P^{s}$ of this equation are piecewise constant solutions, where $P^{s}$ can take only two values $p_{q}^{ \pm}$and $p_{n-q}^{\mp}$. Analysis of the corresponding discrete energy functional

$$
\mathcal{E}_{d}(P):=\frac{1}{2} \sum_{i=1}^{N} \log \left(1+p_{i}^{2}\right)
$$

shows the following.

(1) For any $M$ (the mean density) there are at most two asymptotically stable steady states, one of which is spatially uniform.

(2) The uniform solution is a local minimum (maximum) of $\mathcal{E}_{d}$ when $M<1$ $(M>1)$.

(3) When it exists, the single peak solution is a local minimizer of $\mathcal{E}_{d}$. When $M>1$ it is the only minimizer and hence attracts the flow for almost all initial data.

Here a 'single-peak' solution is one for which most of the mass is concentrated on one lattice site. These results show that on any finite-dimensional lattice the only stable solutions are the single-peak solution and possibly the uniform solution, and they show why one expects either a numerical blowup, as in Figure 1 (d), or convergence to a uniform solution for the PDE.

For the continuum problem we proceed as in the discrete problem, albeit formally since the existence of a minimizer is not proven. We first calculate the variational equation for $\mathcal{E}_{c}$ (note that $\mathcal{E}_{c}$ is continuously Fréchet-differentiable in $L^{1}(\Omega)$ ). In view of the mass constraint, the Lagrange multiplier rule implies that there exists a parameter $\lambda \in \mathbb{R}$ such that a critical point $p \in \mathcal{D}$ satisfies the equation

$$
\int_{\Omega}[\mathcal{T}(p) p-\lambda] \varphi d x=\int_{\Omega}\left[\frac{p}{1+p^{2}}-\lambda\right] \varphi d x=0
$$

for all test functions $\varphi \in C^{\infty}(\Omega)$. According to the Fundamental Lemma of the calculus of variations [3, Folgerung 2.11, page 75], a critical point of the minimizing problem (3.14) solves the Euler-Lagrange equation

$$
\mathcal{T}(p) p=\frac{p}{1+p^{2}}=\lambda
$$


almost everywhere in $\Omega$. This is equivalent to stating that $\mathcal{T}(p)$ is proportional to the eigenfunction $\Psi \equiv 1$ of $d^{2} / d x^{2}$ with Neumann data. Thus critical points of the minimizing problem (3.14) must be piecewise-constant functions taking either of the values $p^{*}(\lambda)$ and $p_{*}(\lambda)=1 / p^{*}$, with $p_{*}(\lambda) \leq 1 \leq p^{*}(\lambda)$ where the $\lambda$ in the argument indicates the dependence of these values on the Lagrange multiplier. By integrating (3.18) one finds that $\lambda \in\left(0, M /\left(1+M^{2}\right)\right] \subset(0,1 / 2]$, and for every $\lambda$ in this interval there is a corresponding critical point of the constrained energy functional. Since $\mathcal{T}(p) p$ is continuous at jumps in $p$, the corner conditions which demand that $\Phi(p)-\lambda p$ be continuous at jumps are automatically satisfied.

The solutions of (3.18) are

$$
p^{*}(\lambda)=\frac{1}{2 \lambda}+\sqrt{\frac{1}{4 \lambda^{2}}-1}, \quad p^{*}(\lambda)+p_{*}(\lambda)=\lambda^{-1} \quad \text { and } \quad p^{*}(\lambda) \cdot p_{*}(\lambda)=1 .
$$

Let $\omega_{1}$ be the fraction of $\Omega$ where $p=p^{*}(\lambda)$ and let $\omega_{2}=1-\omega_{1}$ be the fraction of $\Omega$ where $p=p_{*}(\lambda)$. Then $p^{*} \omega_{1}+p_{*} \omega_{2}=M, \omega_{1}=\left(M-p_{*}\right) /\left(p^{*}-p_{*}\right)$, and $\omega_{2}=\left(M-p^{*}\right) /\left(p_{*}-p^{*}\right)$. The unique spatially uniform solution that exists for all $M \in(0, \infty)$ is $p^{*}=1 / M, p_{*}=M, \omega_{1}=0, \omega_{2}=1, \lambda=M /\left(1+M^{2}\right)$. Note that $p^{*} \rightarrow \infty$ and $p_{*} \rightarrow 0$ as $\lambda \rightarrow 0$

The variational problem (3.14) now reads

$$
\inf _{0<\lambda \leq \frac{M}{1+M^{2}}} \hat{\mathcal{E}}(\lambda)=\inf _{0<\lambda \leq \frac{M}{1+M^{2}}} \frac{|\Omega|}{2} \log \left(\left(1+\left(p^{*}\right)^{2}\right)^{\omega_{1}}\left(1+p_{*}^{2}\right)^{\omega_{2}}\right),
$$

where $\hat{\mathcal{E}}(\lambda) \equiv \mathcal{E}_{c}(p(\lambda))$. The first derivative of $\hat{\mathcal{E}}(\lambda)$ with respect to $\lambda$ is

$$
\begin{aligned}
\frac{\partial}{\partial \lambda} \hat{\mathcal{E}}(\lambda)= & \frac{4|\Omega|(2 \lambda-M)}{\sqrt{1-4 \lambda^{2}}\left(2\left(1+\sqrt{1-4 \lambda^{2}}\right)\right)\left(4 \lambda^{2}-1-\sqrt{1-4 \lambda^{2}}\right)^{2}} \\
& \times\left[2+8 \lambda^{4}-10 \lambda^{2}+\left(2-6 \lambda^{2}\right) \log 2\right. \\
& +\left(2 \lambda^{2} \sqrt{1-4 \lambda^{2}}-2 \sqrt{1-4 \lambda^{2}}\right) \log \left(1+\sqrt{1-4 \lambda^{2}}\right) \\
& -\left(6 \lambda^{2}-2+2 \lambda^{2} \log 2+2 \log 2\right) \sqrt{1-4 \lambda^{2}} \\
& -\left(\lambda^{2} \sqrt{1-4 \lambda^{2}}-\sqrt{1-4 \lambda^{2}}+3 \lambda^{2}-1\right) \log \left(\lambda^{2}\right) \\
& \left.+\left(6 \lambda^{2}-2\right) \log \left(1+\sqrt{1-4 \lambda^{2}}\right)\right] .
\end{aligned}
$$

and from this we see that the first factor vanishes at $\lambda=M / 2$, and second factor vanishes at $\lambda=0.5$. The latter is not attainable for $M \neq 1$ with $M<\infty$, and therefore $\hat{\mathcal{E}}(\lambda)$ has a unique critical point at $\lambda=M / 2$. Note that $M / 2 \in(0, M /(1+$ $\left.\left.M^{2}\right)\right]$ if $M \leq 1$, but $M / 2 \notin\left(0, M /\left(1+M^{2}\right)\right]$ if $M>1$, and thus $\hat{\mathcal{E}}(\lambda)$ has a critical 
point only when $M<1$. From the expression for $\hat{\mathcal{E}}(\lambda)$ we see that

$$
\begin{aligned}
\frac{2 \hat{\mathcal{E}}(\lambda)}{|\Omega|}= & \log \left(1+\left(\frac{1}{2 \lambda}+\sqrt{\frac{1}{4 \lambda^{2}}-1}\right)^{2}\right)^{\frac{\lambda\left(M+M \sqrt{1-4 \lambda^{2}}-2 \lambda\right)}{1+\sqrt{1-4 \lambda^{2}}-4 \lambda^{2}}} \\
& +\log \left(1+\left(\frac{2 \lambda 1}{1+\sqrt{1-4 \lambda^{2}}}\right)^{2}\right)^{\frac{\left(1+\sqrt{1-4 \lambda^{2}}-2 M \lambda\right)\left(1+\sqrt{1-4 \lambda^{2}}\right)}{1+\sqrt{1-4 \lambda^{2}}-4 \lambda^{2}}} .
\end{aligned}
$$

Therefore

$$
\lim _{\lambda \rightarrow 0} \log \left(1+\left(\frac{1}{2 \lambda}+\sqrt{\frac{1}{4 \lambda^{2}}-1}\right)^{2}\right)^{\frac{\lambda\left(M+M \sqrt{1-4 \lambda^{2}}-2 \lambda\right)}{1+\sqrt{1-4 \lambda^{2}}-4 \lambda^{2}}}=0
$$

and

$$
\lim _{\lambda \rightarrow 0} \log \left(1+\left(\frac{2 \lambda}{1+\sqrt{1-4 \lambda^{2}}}\right)^{2}\right)^{\frac{\left(1+\sqrt{1-4 \lambda^{2}}-2 M \lambda\right)\left(1+\sqrt{1-4 \lambda^{2}}\right)}{1+\sqrt{1-4 \lambda^{2}}-4 \lambda^{2}}}=0
$$

Consequently $\lim _{\lambda \rightarrow 0} \hat{\mathcal{E}}(\lambda)=0$, and

$$
\hat{\mathcal{E}}\left(\frac{M}{1+M^{2}}\right)=\mathcal{E}_{c}(M)=\frac{|\Omega|}{2} \log \left(1+M^{2}\right)>\hat{\mathcal{E}}(\varepsilon)
$$

for sufficiently small $\varepsilon>0$.

Thus $\hat{\mathcal{E}}(\lambda)$ is monotone increasing on the interval $(0, M / 2]$ and monotone decreasing on $\left(M / 2, M /\left(1+M^{2}\right)\right]$ if $M<1$, while $\hat{\mathcal{E}}(\lambda)$ is monotone increasing on $\left(0, M /\left(1+M^{2}\right)\right]$ if $M>1$. Since $p^{*} \rightarrow \infty$ and $p_{*} \rightarrow 0$ as $\lambda \rightarrow 0$, we conclude that as $\lambda \rightarrow 0$ the 'least energy solution' for the continuum case approaches a distribution that is zero almost everywhere in $\Omega$ and equal to $\infty$ on a set of zero measure, i.e., a single $\delta$-function. In [22] we reached a similar conclusion beginning from the discretized version of the problem.

\subsection{The shape of the minimizers in a regularized problem}

To further understand the qualitative behavior of the least energy solution of (3.1) or more precisely, the critical point for the minimizing problem (3.14), we consider the following Cahn-Hilliard regularization of the minimizing problem. ${ }^{2}$

$$
\min _{p \in \mathcal{D}_{0}} \int_{\Omega} \gamma^{2}\left|p_{x}\right|^{2}+\frac{1}{2} \log \left(1+p^{2}\right) d x
$$

2 The corresponding evolution equation will be treated later. 
Here the energy functional is defined on a set of smoother functions

$$
\mathcal{D}_{0}:=\left\{p \in H^{1}(\Omega)\left|p \geq 0, \int_{\Omega} p(x) d x=M\right| \Omega \mid\right\} .
$$

and jumps in the density are not allowed, but $p$ may vary rapidly over small intervals. Such changes are penalized in the energy by the term $\gamma p_{x}^{2}$. The existence of a minimizer of (3.26) follows by the direct method of the calculus of variations, and it follows from elliptic regularity theory (see for instance [14]) that this minimizer satisfies the Euler-Lagrange equation

$$
\left.\begin{array}{rlrl}
2 \gamma^{2} p_{x x} & =\frac{p}{1+p^{2}}-\lambda_{\gamma}, & & \text { in } \Omega \\
p_{x} & =0, & & \text { on } \partial \Omega \\
\int_{\Omega} p(x) & =M|\Omega|=\int_{\Omega} p_{0}(x) d x, &
\end{array}\right\}
$$

where as before, $\lambda_{\gamma} \leq \frac{M}{1+M^{2}}$. Now we assume without loss of generality that $\Omega=(-1,1)$, and following [6], we define $x=\gamma s, z(s)=p(\gamma s)$. Then we can write $(3.28)$ as

$$
\begin{aligned}
2 z^{\prime \prime} & =\frac{z}{1+z^{2}}-\lambda, \quad \text { in }\left(-\gamma^{-1}, \gamma^{-1}\right) \\
z^{\prime}\left( \pm \gamma^{-1}\right) & =0 \\
\int_{\Omega_{\gamma}} p(s) d s & =2 \gamma^{-1} M .
\end{aligned}
$$

Any solution of this problem gives rise to a trajectory in the $\left(z, z^{\prime}\right)$ phase plane which begins and ends on the $z$ axis and has duration $2 \gamma^{-1}$. Furthermore we see that non-constant solutions of (3.29) lie on closed orbits and, thus, they are either monotonic or periodic.

In the new variables problem (3.26) becomes

$$
\min _{z \in \mathcal{D}_{\gamma}} \mathcal{E}_{\gamma}(z):=\min _{z \in \mathcal{D}_{\gamma}} \gamma \int_{-\gamma^{-1}}^{\gamma^{-1}}\left|z^{\prime}\right|^{2} d s+\frac{\gamma}{2} \int_{-\gamma^{-1}}^{\gamma^{-1}} \log \left(1+z^{2}\right) d s,
$$

where

$$
\mathcal{D}_{\gamma}:=\left\{z \in H^{1}(\Omega) \mid \int_{\Omega} z(s) d s=2 \gamma^{-1} M\right\} .
$$

Following [28], we say that a function $u$ is a relative minimizer of (3.30) if there is 
a neighborhood $\mathcal{N}$ of $u$ in $\mathcal{D}_{\gamma}$ such that

$$
\gamma \int_{-\gamma^{-1}}^{\gamma^{-1}}\left|u^{\prime}\right|^{2} d s+\frac{\gamma}{2} \int_{-\gamma^{-1}}^{\gamma^{-1}} \log \left(1+u^{2}\right) d s \leq \gamma \int_{-\gamma^{-1}}^{\gamma^{-1}}\left|v^{\prime}\right|^{2} d s+\frac{\gamma}{2} \int_{-\gamma^{-1}}^{\gamma^{-1}} \log \left(1+v^{2}\right) d s
$$

for all $v \in \mathcal{N} \cap \mathcal{D}_{\gamma}$. If the above relation holds for all $v \in \mathcal{D}_{\gamma}$ then $u$ is called an absolute minimizer. The next result shows that non-monotonic solutions of (3.29) are not absolute or relative minimizers of (3.30).

Theorem 3.4 Relative minimizers of (3.30) are either constant or monotonic.

Proof: We assume that there is a non-monotonic relative minimizer of (3.30) and we denote that minimizer by $u$. The second variation of $\mathcal{E}_{\gamma}$ at $u$ is given by

$$
\left.\frac{1}{\gamma} \frac{d^{2}}{d \xi^{2}} \mathcal{E}_{\gamma}(u+\xi \eta)\right|_{\xi=0}=\int_{-\gamma^{-1}}^{\gamma^{-1}} 2\left(\eta^{\prime}\right)^{2}+\frac{\left(1-u^{2}\right)}{\left(1+u^{2}\right)^{2}} \eta^{2} d s
$$

where $\eta \in H^{1}\left(-\gamma^{-1}, \gamma^{-1}\right)$ and $\int_{-\gamma^{-1}}^{\gamma^{-1}} \eta d s=0$. Since non-constant solutions of (3.29) lie on closed orbits and $u$ is non-monotonic, there is an $S \in\left(-\gamma^{-1}, \gamma^{-1}\right)$ such that $u(S)=u\left(-\gamma^{-1}\right), u^{\prime}(S)=0\left(=u^{\prime}\left(-\gamma^{-1}\right)\right)$. Now let

$$
\eta_{0}= \begin{cases}u^{\prime}(s), & -\gamma^{-1} \leq s \leq S \\ 0, & S \leq s \leq \gamma^{-1}\end{cases}
$$

and $\eta_{1}(s) \in H^{1}\left(-\gamma^{-1}, \gamma^{-1}\right)$ satisfying $\int_{-\gamma^{-1}}^{\gamma^{-1}} \eta_{1} d s=0$ and $\eta_{1}\left(-\gamma^{-1}\right)=1, \eta_{1}(s)=0$ for $S \leq s \leq \gamma^{-1}$. Set

$$
\eta(s)=\eta_{0}(s)+\chi \eta_{1}(s)
$$

with a constant $\chi$ that we specify later. We see that $\eta \in H^{1}\left(-\gamma^{-1}, \gamma^{-1}\right)$ and $\int_{-\gamma^{-1}}^{\gamma^{-1}} \eta d s=0$ holds and

$$
\begin{aligned}
\left.\frac{1}{\gamma} \frac{d^{2}}{d \xi^{2}} \mathcal{E}_{\gamma}(u+\xi \eta)\right|_{\xi=0}= & \int_{-\gamma^{-1}}^{S} 2\left(u^{\prime \prime}\right)^{2}+\frac{\left(1-u^{2}\right)}{\left(1+u^{2}\right)^{2}}\left(u^{\prime}\right)^{2} d s \\
& +2 \chi \int_{-\gamma^{-1}}^{S} 2 u^{\prime \prime} \eta_{1}^{\prime}+\frac{\left(1-u^{2}\right)}{\left(1+u^{2}\right)^{2}} u^{\prime} \eta_{1} d s+O\left(\chi^{2}\right)
\end{aligned}
$$


Using the fact that $u$ is a solution of (3.29) we see that

$$
2 u^{\prime \prime \prime}=\frac{1-u^{2}}{\left(1+u^{2}\right)^{2}} u^{\prime}
$$

If we now replace $\frac{1-u^{2}}{\left(1+u^{2}\right)^{2}} u^{\prime}$ by $2 u^{\prime \prime \prime}$ in (3.33) and integrate by parts we see that

$$
\left.\frac{1}{\gamma} \frac{d^{2}}{d \xi^{2}} \mathcal{E}_{\gamma}(u+\xi \eta)\right|_{\xi=0}=-4 \chi u^{\prime \prime}\left(-\gamma^{-1}\right)+O\left(\chi^{2}\right)
$$

Since $u$ is not constant we know that $u^{\prime \prime}\left(-\gamma^{-1}\right) \neq 0$ and we can choose an appropriate $\chi$ such that

$$
\left.\frac{1}{\gamma} \frac{d^{2}}{d \xi^{2}} \mathcal{E}_{\gamma}(u+\xi \eta)\right|_{\xi=0}<0
$$

which contradicts our assumption that $u$ is a relative minimizer.

If a relative minimizer is constant, it must be equal to $M$ to satisfy the constraint. However for sufficiently small $\gamma$ the constant solution is not the absolute minimizer of the variational problem (3.26), and thus the absolute minimizer must be strictly monotonic. If we denote by $\left(p_{\gamma}\right)_{\gamma>0}$ the sequence of absolute minimizers of (3.26), we see that as $\gamma \rightarrow 0$ the limit $p^{\star}$ is also monotonic. This limit is in the sense of measure, since according to [27, page 571] there exists a subsequence of the $\left(p_{\gamma}\right)$, for simplicity again denoted by $\left(p_{\gamma}\right)$, such that

$$
\int_{-1}^{1} p_{\gamma} \phi d x \rightarrow\langle\mu, \phi\rangle
$$

for every bounded $\phi \in C(-1,1)$ as $\gamma \rightarrow 0$, where $\mu$ is a Radon measure on $(-1,1)$. In view of the previous analysis we see that the measure $\mu$ is a $\delta$-distribution, concentrated on boundary points of the interval $[-1,1]$. We can also use these conclusions to describe the shape of the least energy solution when we restrict ourselves to periodic solutions. In particular, we see that the number of peaks of the least energy solution is given by the periodicity of the class of functions that we study, as can be seen by using a reflection argument and repeating the preceding arguments on the subinterval $\left[1-\frac{2}{l}, 1\right]$, where $l$ denotes the period of the functions considered.

\subsection{Finite time blowup of weak solutions}

We now return to the time dependent problem, and define a weak solution of (3.1) as follows. 
Definition 3.5 A function $p(x, t)$ with

$$
p \in L^{\infty}\left(L_{+}^{\infty}(\Omega) ;(0, T)\right) \cap L^{2}\left(H^{1}(\Omega) ;(0, T)\right), p_{t} \in L^{2}\left(\left(H^{1}(\Omega)\right)^{*} ;(0, T) ;\right)
$$

is called a weak solution of (3.1) if for all $h \in L^{2}\left(H^{1}(\Omega) ;(0, T)\right)$ the following identity holds:

$$
0=\int_{0}^{T}\left\langle p_{t}, h\right\rangle d t+\int_{0}^{T} \int_{\Omega}(\mathcal{T}(p) p)_{x} h_{x} d x d t .
$$

$\left(\right.$ Here $\langle\cdot, \cdot\rangle$ denotes the dual product between $H^{1}(\Omega)$ and its dual space $\left.\left(H^{1}(\Omega)\right)^{*}\right)$.)

Even though we have no rigorous existence result in the case of supercritical initial data, let us assume that a weak solution of (3.1) exists locally, and formulate a finite time blow-up result for such solutions of (3.1) in the case of one space dimension.

Theorem 3.6 Let $\Omega \subset \mathbb{R}$, and suppose that for sufficiently smooth initial data $p_{0}(x)$ there exists a weak solution of (3.1) locally in time. Furthermore, assume that

$$
\mathcal{E}_{c}\left(p_{0}\right)<\mathcal{E}_{c}(M)
$$

where $M$ is the mean density of $p_{0}(x)$. Then the weak solution of (3.1) must blow up in finite time, i.e., there exists a maximal time $T_{\max }<\infty$ such that $\|p(x, t)\|_{H^{1}(\Omega)} \rightarrow \infty$ as $t \rightarrow T_{\max }$.

Proof of Theorem 3.6: The proof is a simple consequence from the properties of the functional $\mathcal{E}_{c}(t)$ and the Sobolev embedding for $H^{1}(\Omega) \subset C^{\beta}(\bar{\Omega})$ with $\beta \in$ $(0,1)$. First, we recall that $\mathcal{E}_{c}(t)$ is bounded below. If we suppose that a weak solution of (3.1) exists, we can multiply (3.1) with $\mathcal{T}(p) p \in L^{2}\left((0, T) ; H^{1}(\Omega)\right)$ and integrate the equation by parts over $\Omega$. From this we see that

$$
\frac{d}{d t} E_{c}(t)=-\int_{\Omega}\left|(\mathcal{T}(p) p)_{x}\right|^{2} d x
$$

also holds for a weak solution, not only for classical solutions, of (3.1). If we now assume that $T_{\max }=\infty$ one can conclude that

$$
\lim _{t \rightarrow \infty} \int_{\Omega}\left|(\mathcal{T}(p(x, t)) p(x, t))_{x}\right|^{2} d x=0 .
$$

This implies that $\lim _{t \rightarrow \infty} \mathcal{T}(p(x, t)) p(x, t)=$ const a.e. in $\Omega$. Since $p \in H^{1}(\Omega)$, $\mathcal{T}(p) p \in H^{1}(\Omega)$ and as a consequence of the Sobolev embedding theorem, $[15,1]$ $\mathcal{T}(p) p$ is continuous and $\lim _{t \rightarrow \infty} \mathcal{T}(p(x, t)) p(x, t)=$ const in $\Omega$. It follows that $\lim _{t \rightarrow \infty} p_{t}(x, t)=0$, and therefore $\mathcal{T}(p(x, t)) p(x, t)$ must converge to an eigenfunction of the Laplacian with homogeneous Neumann boundary conditions, i.e., 
to a constant. But this implies that $p(x, t)$ must converge to a constant and consequently it converges to $M$ as $t \rightarrow \infty$. However, the fact that $\hat{\mathcal{E}}$ is non-increasing along orbits, together with the assumption (3.37), renders this impossible

We can now begin to synthesize our understanding of the temporal evolution of solutions for various initial data. We know that for subcritical initial data the solution exists globally in time and converges to the constant solution $p=M$. Thus the energy of such initial data must be greater than that of the uniform solution, which is $\mathcal{E}_{c}(M)=|\Omega| \log \left(1+M^{2}\right) / 2$. If $M>1$ a linear analysis shows that the constant solution is unstable. In Figure 1 (c) and (d) one sees that having supercritical initial data is not sufficient to guarantee the blow-up of the $H^{1}$-norm of the solution, but the energy condition (3.37) provides a sufficient condition. In particular, we know from section 3.2 that if $M<1$ there is a $\lambda_{1} \in(0,(M / 1+$ $\left.M^{2}\right)$ ] such that $\hat{\mathcal{E}}\left(\lambda_{1}\right)=\mathcal{E}_{c}(M)$. In this case small perturbations of step-functions satisfying

$$
\frac{p}{1+p^{2}}=\lambda<\lambda_{1}
$$

lead to finite time blow-up of the solution, while step-functions satisfying

$$
\frac{p}{1+p^{2}}=\lambda>\lambda_{1}
$$

can either lead to blow-up in finite time or convergence to the uniform distribution. ${ }^{3}$ However it is extremely complicated to calculate the dependence of $\lambda_{1}$ on $M$ and 1, as we see from formula (3.22).

Since critical points exist for any $\lambda \in\left(0, M /\left(1+M^{2}\right]\right.$, one expects that the evolution will in general exhibit a complicated coarsening process en route to the minimum energy solution, as was seen earlier for the finite-dimensional problem (cf. Figure 3 in [22]). The evolution decreases the energy, but solutions may pass near critical points and be transiently 'trapped' in the neighborhood of a critical point near its stable manifold, only to eventually leave in the direction of the unstable manifold. As long as it exists the solution continues to decrease the energy, although not necessarily by steepest descent, and may again be transiently trapped near a critical point having fewer maxima (we have seen in the discrete case that the energy decreases with a decreasing number of peaks of the steady state (see [22]). However the least energy solution of (3.14) is expected to be a $\delta$-function concentrated either at the boundary, or for symmetric solutions, at the center of the interval, and our numerical computations confirm this expectation. A simulation demonstrating the former case is shown in Figure 2, while the latter is shown in Figure 3. However in the discrete formulation the localization of the peak

3 Our numerical experiments suggest that the $L^{\infty}$-norm of $p(x, t)$ blows up in finite time, but the theorem only gives us the finite time blowup in the $H^{1}$-norm. 
at the boundary is not necessary since every single-peaked steady state solution has the same energy.
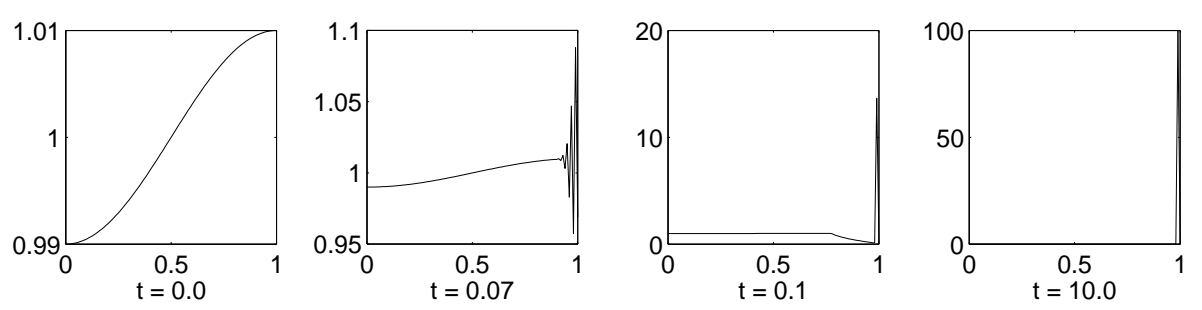

FiguRE 2. Blow-up at the boundary for initial data $p(x, 0)=1.0-0.01 \cos (\pi x)$, and $M=1$. Simulations use 101 lattice points.
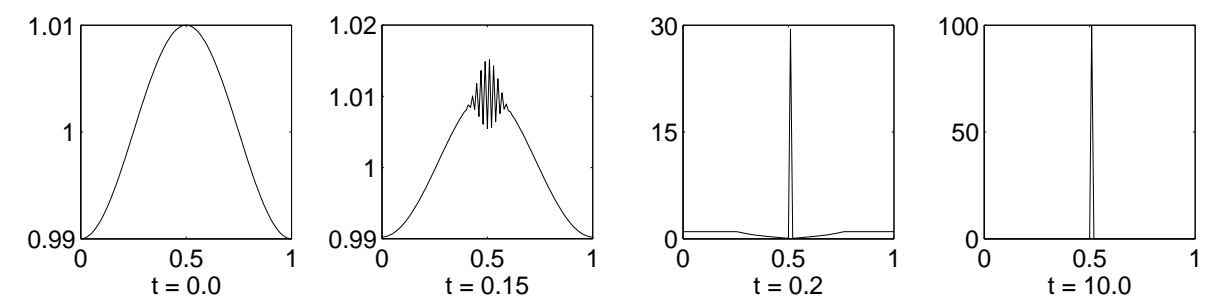

FIgURE 3. Interior blow-up for initial data $p(x, 0)=1.0-0.01 \cos (2 \pi x)$, and $M=1$. Simulation data as above.

\section{The Cahn-Hilliard evolution}

The gradient flow for the regularized energy functional (3.26) is the Cahn-Hilliardlike equation

$$
\left.\begin{array}{rlrl}
\frac{\partial p_{\gamma}}{\partial t} & =\Delta\left(\frac{p_{\gamma}}{1+p_{\gamma}^{2}}\right)-2 \gamma^{2} \Delta^{2} p_{\gamma}, & & \text { in } Q_{T} \\
\frac{\partial}{\partial \nu}\left(\frac{p_{\gamma}}{1+p_{\gamma}^{2}}\right) & =0, \frac{\partial}{\partial \nu}\left(\Delta p^{\gamma}\right)=0, & & \text { on } \Gamma_{T} \\
p^{\gamma}(x, 0) & =p_{0}(x), & & \text { in } \Omega .
\end{array}\right\}
$$

The local existence of a weak solution of this problem for every $p_{0} \in H^{1}(\Omega)$ can be shown for $N \leq 3$ by using a Galerkin approximation for the system

$$
\left.\begin{array}{rlrl}
u_{t} & =\Delta w, & & \text { in } Q_{T} \\
w & =\frac{u}{1+u^{2}}-2 \gamma^{2} \Delta u, & & \text { in } Q_{T} \\
\frac{\partial w}{\partial \nu} & =0=\frac{\partial w}{\partial \nu}, & & \text { on } \Gamma_{T} \\
u(x, 0) & =p_{0}(x), & & \text { in } \Omega .
\end{array}\right\}
$$


This can be proven by a slight modifications of the method used for the standard Cahn-Hilliard equation in [8]. The result is that there exists a weak solution of (4.2) such that $u \in L^{\infty}\left(H^{1}(\Omega) ;(0, T)\right), w \in L^{2}\left(H^{1}(\Omega) ;(0, T)\right)$ and $u_{t} \in L^{2}\left(\left(H^{1}(\Omega)\right)^{*} ;(0, T)\right)$ such that

$$
\left.\begin{array}{rlrl}
\int_{\Omega} u_{t} \varphi d x & =-\int_{\Omega} w_{x} \varphi_{x}, & & \forall \varphi \in H^{1}(\Omega) \\
\int_{\Omega} w \varphi & =\int_{\Omega} \frac{u}{1+u^{2}} \varphi+2 \gamma^{2} u_{x} \varphi_{x}, & \forall \varphi \in H^{1}(\Omega) \\
u(0) & =p_{0}(x) \in H^{1}(\Omega) . &
\end{array}\right\}
$$

The existence of the Lyapunov function
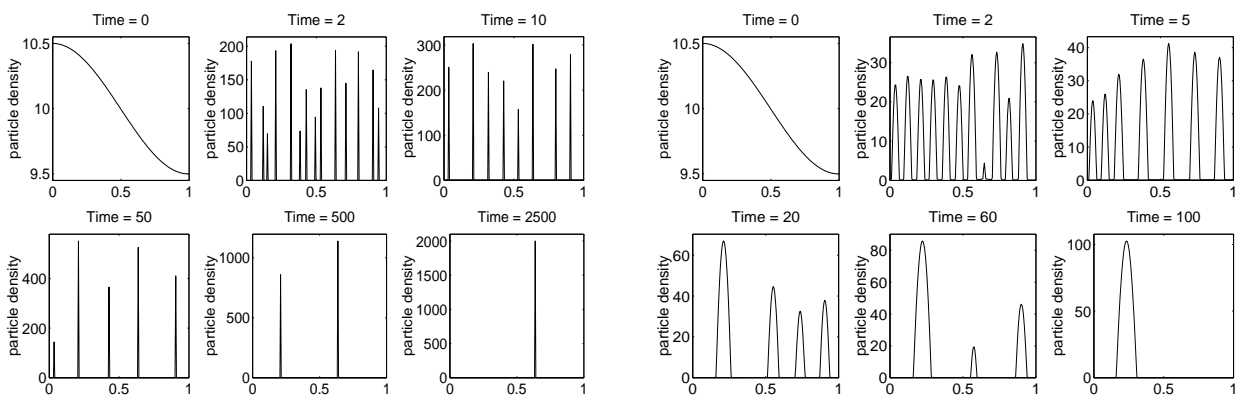

FigURE 4. The coarsening effect for the singular model (left) and the "Cahn-Hilliard" approximation (right). In both simulations, we used initial data $p(x, 0)=10.0+0.5 \cos (\pi x)$ with $1=1$ and 201 lattice points. For the Cahn-Hilliard approximation, $\gamma$ was set at $10^{-6}$

$$
\int_{\Omega} \gamma^{2}\left|p_{x}\right|^{2}+\frac{1}{2} \log \left(1+p^{2}\right) d x
$$

implies that the solution exists globally in time and converges to a stationary solution of (4.1), at least for a subsequence $\left(t_{k}\right)_{k \in \mathbb{N}}$ with $t_{k} \rightarrow \infty$. Thus solutions cannot blow up, but a comparison of the numerical results for the time asymptotics of the "Cahn-Hilliard" approximation with the numerical results for the singular problem, shows that both systems exhibit the coarsening effect, and the final steady state solutions of the "Cahn-Hilliard" approximation have similar qualitative properties to the blow-up profile of the singular model. Smaller values of $\gamma$ in the Cahn-Hilliard problem of course result in "sharper" aggregations.

However, the "Cahn-Hilliard" approach can not be derived directly from the lattice walk. The fourth order approximation obtained by retaining the next term in a Taylor expansion (using suitable scalings) is

$$
p_{t}=(T(p) p)_{x x}+\gamma(T(p) p)_{x x x x} .
$$


However, the fourth order term has the wrong sign where $p(x, t)$ is subcritical and the right sign where it is supercritical. Numerical simulations of this fourth order equation and its effects are carried out in detail in [23]. It is known that if nonlocal effects are included in the flux, rather than directly in the master equation, a Cahn-Hilliard-like equation is obtained [18].

\section{Analysis of the non-singular problem}

In the derivation of the singular model we assumed that the control substance evolves on a faster time scale than the jump process, but if they occur on comparable scales we obtain the following strongly coupled reaction-diffusion system consisting of a nonlinear parabolic partial differential equation and an ordinary differential equation.

$$
\left.\begin{array}{rlrl}
p_{t} & =(\mathcal{T}(w) p)_{x x}, & & \text { in } Q_{T} \\
\epsilon w_{t} & =p^{2}-\mu w, & & \text { in } Q_{T} \\
(\mathcal{T}(w) p)_{x} & =0, & & \text { on } \Gamma_{T} \\
p(x, 0) & =p_{0}(x), w(x, 0)=w_{0}(x), & & \text { in } \Omega .
\end{array}\right\}
$$

Here we use the form $\mathcal{T}(w)=1 /(1+w)$ for the transition rates. Our main goal in this section is to establish the local existence in time of a solution of this system. In effect, we show that incorporating the finite evolution rate of $w$ provides a different regularization of the problem. As we showed in [23], one can expect that the asymptotic behavior of solutions to this regularized problem is similar to the asymptotic behavior of the solution to (3.1).

To prove the local existence of a classical solution for this model, we introduce the new function

$$
u(x, t)=\frac{p(x, t)}{1+w(x, t)} .
$$

We set $\mu=1$ hereafter and thus obtain the problem

$$
\left.\begin{array}{rlrl}
u_{t} & =\frac{u_{x x}-w_{t} u}{1+w}=\frac{u_{x x}}{1+w}-\frac{1}{\epsilon}(1+w) u^{3}+\frac{w u}{\epsilon(1+w)}, & & \text { in } Q_{T} \\
\epsilon w_{t} & =(1+w)^{2} u^{2}-w, & & \text { in } Q_{T} \\
u_{x} & =0, & & \text { on } \Gamma_{T} \\
u(x, 0) & =\frac{p_{0}(x)}{1+w_{0}(x)}, w(x, 0)=w_{0}(x), & & \text { in } \Omega .
\end{array}\right\}
$$

Under this transformation we lose the higher order terms in $w$ in the first equation, but pay for this simplification by obtaining a nonlinear equation for $w$. Since we are looking for positive solutions of (5.1) resp. (5.2) the first equation of (5.2) 
implies that for such a solution

$$
\int_{\Omega} u(x, t) d x \leq \text { const } \forall t \geq 0 .
$$

This inequality follows since the first equation can be written as $u_{t}+u_{t} w+w_{t} u=$ $u_{x x}$. Thus we see that

$$
\int_{\Omega} u_{t}+(w u)_{t} d x=0
$$

If we integrate the equation over $(0, T)$, we see that for non-negative solutions

$$
\int_{\Omega} u(x, t) d x \leq \int_{\Omega}(u(x, 0)+w(x, 0) u(x, 0)) d x .
$$

Thus the $L^{1}$-norm of a non-negative solution $u$ is uniformly bounded for all $t \geq 0$. For the remainder of this section we set $\epsilon=1$, but the existence result and all other statements can be shown to be true for every $\varepsilon>0$ by making the corresponding changes.

Theorem 5.1 Suppose that $u_{0}(x)$ belongs to the class $C^{2, \beta}(\Omega)(\beta \in(0,1))$ satisfying $u_{x}(x, 0)=0$ on $\partial \Omega$ and $w_{0}(x) \in H^{2}(\Omega)$. Furthermore, assume that $u_{0}(x)$ and $w_{0}(x)$ are positive in $\Omega$. Then there is a time $T>0$ such that there exists a solution $(u(x, t), w(x, t))$ of (5.2) with

$$
u \in L^{\infty}\left(L_{+}^{\infty}(\Omega) ;(0, T)\right) \cap L^{2}\left(H^{2}(\Omega) ;(0, T)\right), u_{t} \in L^{2}\left(Q_{T}\right)
$$

and $w \in L^{\infty}\left(L_{+}^{\infty}(\Omega) ;(0, T)\right) \cap L^{2}\left(H^{2}(\Omega) ;(0, T)\right), w_{t} \in L^{2}\left(H^{2}(\Omega) ;(0, T)\right)$.

Furthermore $u(x, t)$ belongs to $C^{1+\frac{\beta}{2}, 2+\beta}\left(\overline{Q_{T}}\right), u(x, t) \geq 0$ and $w(x, t) \geq 0$ for all $t \in(0, T)$.

Proof: We prove the local (in time) existence of a solution of (5.2) by applying Schauder's fixed point theorem (see for instance [11]. Therefore we define the mapping

$$
\begin{array}{ccc}
A: L^{2}\left(H^{2}(\Omega) ;(0, T)\right) & \rightarrow & L^{2}\left(H^{2}(\Omega) ;(0, T)\right) \\
f & \mapsto & u=: A f
\end{array}
$$

where $u$ is the solution of

$$
\left.\begin{array}{rlrl}
u_{t} & =\frac{u_{x x}}{1+w}-(1+w) u^{3}+\frac{w u}{1+w}, & & \text { in } Q_{T} \\
w_{t} & =(1+w)^{2} f^{2}-w, & & \text { in } Q_{T} \\
u_{x}(x, t) & =0, & & \text { on } \Gamma_{T} \\
u(x, 0) & =\frac{p_{0}(x)}{1+w_{0}(x)}, w(x, 0)=w_{0}(x), & & \text { in } \Omega .
\end{array}\right\}
$$


For $f, w_{0} \in H^{2}(\Omega)\left(f \in L^{2}\left(H^{2}(\Omega) ;(0, T)\right)\right)$, the second equation is an ODE in the Banach space $H^{2}(\Omega)$. There exists a solution of this ODE and we can assume that for a sufficiently small time $T_{0}$

$$
\sup _{0 \leq t \leq T_{0}}\|w(x, t)\|_{H^{2}(\Omega)} \leq C\left(\|f(x, t)\|_{L^{2}\left(H^{2}(\Omega) ;\left(0, T_{0}\right)\right)},\left\|w_{0}(x)\right\|_{H^{2}(\Omega)}, T_{0}\right) .
$$

Thus we can assume that

$$
\sup _{0 \leq t \leq T_{0}}\|w(x, t)\|_{C^{1}(\bar{\Omega})} \leq C\left(\|f(x, t)\|_{L^{2}\left(H^{2}(\Omega) ;\left(0, T_{0}\right)\right)},\left\|w_{0}(x)\right\|_{H^{2}(\Omega)}, T_{0}\right) .
$$

Since we are in the one dimensional case, the first equation becomes a quasi-linear parabolic equation in $Q_{T}$ with ellipticity function $1 /(1+w(x, t)) \in C^{1, \beta}(\bar{\Omega})$ for all $0 \leq t \leq T_{0}$. From Theorem 7.4 in [15] we conclude that there exists a unique solution $u(x, t) \in C^{1+\frac{\beta}{2}, 2+\beta}\left(\overline{Q_{T_{0}}}\right)$ of the first equation. Thus the mapping $A$ is well-defined. The function $v(x, t)=u_{x}(x, t)$ solves the linear parabolic boundary value problem

$$
\left.v_{t}=a_{1}(w) v_{x x}+a_{2}\left(w, w_{x}\right) v_{x}+a_{3}(u, w) v+a_{4}\left(u, w, w_{x}\right), \text { in } Q_{T},\right\}
$$

at least in a weak sense, wherein

$$
\begin{array}{ll}
a_{1}(w)=\frac{1}{1+w}, & a_{3}(u, w)=\frac{w}{1+w}-3(1+w) u^{2} \\
a_{2}\left(w, w_{x}\right)=-\frac{w_{x}}{(1+w)^{2}}, & a_{4}\left(u, w, w_{x}\right)=-\left(u^{3}-\frac{u}{1+w}+\frac{w u}{(1+w)^{2}}\right) w_{x} .
\end{array}
$$

Using the regularity of the functions $u(x, t)$ and $w(x, t)$ we see that $a_{1}(w)$ is continuous and bounded in $Q_{T}, a_{2}\left(w, w_{x}\right), a_{3}(u, w) \in L^{2}\left(Q_{T}\right)$ satisfy the conditions of Theorem 9.1 in $\left[15\right.$, Chapter IV] and $a_{4}\left(u, w, w_{x}\right) \in L^{2}\left(Q_{T}\right)$. Since $u_{x}(0)=0$ on $\partial \Omega$, the theorem also implies the existence of a unique solution of problem (5.6) belonging to the class $L^{2}\left(H^{2}(\Omega) ;(0, T)\right)$ with $v_{t} \in L^{2}\left(Q_{T}\right)$. Furthermore this solution satisfies the estimate

$$
\begin{aligned}
& \sum_{j=0}^{2} \sum_{2 l+s=j}\left(\int_{0}^{T}\left(\int_{\Omega}\left|\frac{\partial^{l}}{\partial t^{l}} \frac{\partial^{s}}{\partial x^{s}} v(x, t)\right|^{2} d x\right)\right)^{1 / 2} \\
& \leq \text { const }\left(\left\|a_{4}\left(u, w, w_{x}\right)\right\|_{L^{2}\left(Q_{T}\right)}+\|v(x, 0)\|_{H^{1}(\Omega)}\right) .
\end{aligned}
$$

This unique solution coincides with $u_{x}(x, t)$ and we conclude that our solution $u(x, t)$ of problem $(5.2)$ is in fact at least in $L^{2}\left(H^{3}(\Omega) ;(0, T)\right)$.

Let us now turn to the non-negativity of $u(x, t)$ and $w(x, t)$ for positive initial data. The statement for $w(x, t)$ follows directly from solving the second equation of (5.2). To show non-negativity of $u(x, t)$, we multiply the first equation with 
$u^{-}:=\max \{-u, 0\}$ and integrate over $\Omega$. We thus see that:

$$
\begin{aligned}
\frac{d}{d t} \int_{\Omega}\left(u^{-}\right)^{2} d x & =-\int_{\Omega} \frac{\left|u_{x}^{-}\right|^{2}}{1+w} d x+\int_{\Omega} \frac{u^{-} w_{x} u_{x}^{-}}{(1+w)^{2}} d x-\int_{\Omega}(1+w)\left(u^{-}\right)^{4} d x+\int_{\Omega} \frac{w\left(u^{-}\right)^{2}}{1+w} d x \\
& \leq \frac{C\left(\left\|w_{0}(x)\right\|_{H^{2}(\Omega)}, R, T_{0}\right)^{2}}{2} \int_{\Omega} \frac{\left(u^{-}\right)^{2}}{(1+w)^{3}} d x+\int_{\Omega}\left(u^{-}\right)^{2} d x \\
& \leq \max \left\{\frac{C\left(\left\|w_{0}\right\|_{H^{2}(\Omega)}, R, T_{0}\right)^{2}}{2}, 1\right\} \int_{\Omega}\left(u^{-}\right)^{2} d x .
\end{aligned}
$$

Using Gronwall's Lemma [9, Appendix B, p. 624] and the positivity of the initial data, we get that $u(x, t) \geq 0$ for all $t \in\left(0, T_{0}\right)$.

To apply Schauder's fixed point theorem we have to show that the mapping $A$ maps the set $B:=\left\{f:\|f\|_{L^{2}\left(H^{2}(\Omega) ;(0, T)\right)}<R\right\}$ for appropriate $T \leq T_{0}$ and $R$ into itself. Let $f$ be in $\mathrm{B}$ and recall that therefore

$$
\sup _{0 \leq t \leq T}\|w(x, t)\|_{H^{2}(\Omega)} \leq C\left(\left\|w_{0}\right\|_{H^{2}(\Omega)}, R, T\right)
$$

and thus

$$
\sup _{0 \leq t \leq T}\|w(x, t)\|_{C^{1}(\bar{\Omega})} \leq C\left(\left\|w_{0}\right\|_{H^{2}(\Omega)}, R, T\right) .
$$

Multiplying the first equation with $u|u|^{r-1}$ gives us

$$
\begin{aligned}
\frac{1}{r+1} \frac{d}{d t} \int_{\Omega} u^{r+1} d x \leq & -\frac{4 r}{(r+1)^{2}} \int_{\Omega} \frac{\left|\left[u^{\frac{r+1}{2}}\right]_{x}\right|^{2}}{1+w} d x+r \int_{\Omega} \frac{u^{r} u_{x} w_{x}}{(1+w)^{2}} d x+\int_{\Omega} \frac{w u^{r+1}}{1+w} d x \\
\leq & -\frac{2 r}{\left(1+\|w\|_{L^{\infty}(\Omega)}\right)(r+1)^{2}} \int_{\Omega}\left|\left[u^{\frac{r+1}{2}}\right]_{x}\right|^{2} d x \\
& +\frac{r C\left(\left\|w_{x}\right\|_{L^{\infty}(\Omega)}\right)+2}{2} \int_{\Omega} u^{r+1} d x
\end{aligned}
$$

for all $0 \leq t \leq T$. Using Alikakos' method of the Moser iteration (see [2]) we get that

$$
\|u(x, t)\|_{L^{\infty}(\Omega)} \leq C\left(\left\|w_{0}\right\|_{H^{2}(\Omega)}, R, T\right) \max \left\{1,\left\|u_{0}\right\|_{L^{1}(\Omega)},\left\|u_{0}\right\|_{L^{\infty}(\Omega)}\right\}
$$

for all $0 \leq t \leq T$. Thus we have that

$$
\|u\|_{L^{2}\left(L^{\infty}(\Omega) ;(0, T)\right)} \leq C\left(\left\|w_{0}\right\|_{H^{2}(\Omega)}, R, T\right) \max \left\{1,\left\|u_{0}\right\|_{L^{1}(\Omega)},\left\|u_{0}\right\|_{L^{\infty}(\Omega)}\right\} T^{1 / 2} .
$$


Next we obtain an estimate for $\int_{\Omega}\left|u_{x}(x, t)\right|^{2} d x$ for all $0 \leq t \leq T$. We see that:

$$
\begin{aligned}
-\int_{\Omega} u_{t} u_{x x} d x & =-\int_{\Omega} \frac{\left(u_{x x}\right)^{2}}{1+w} d x+\int_{\Omega}\left((1+w) u^{3}-\frac{w u}{1+w}\right) u_{x x} d x \\
& \leq \frac{1}{2} \int_{\Omega}\left((1+w)^{3} u^{6}+\frac{w^{2} u^{2}}{1+w}\right) d x \leq C\left(\left\|w_{0}\right\|_{H^{2}(\Omega)},\left\|u_{0}\right\|_{L^{\infty}(\Omega)}, R, T\right) .
\end{aligned}
$$

Integration of this inequality gives us

$$
\frac{1}{2} \int_{\Omega}\left|u_{x}(x, t)\right|^{2} d x \leq t C\left(\left\|w_{0}\right\|_{H^{2}(\Omega)},\left\|u_{0}\right\|_{L^{\infty}(\Omega)}, R, T\right)+\frac{1}{2} \int_{\Omega}\left|u_{x}(x, 0)\right|^{2} d x
$$

for all $0 \leq t \leq T$ and thus

$$
\left\|u_{x}\right\|_{L^{2}\left(Q_{T}\right)} \leq \sqrt{2} T C\left(\left\|w_{0}\right\|_{H^{2}(\Omega)},\left\|u_{0}\right\|_{L^{\infty}(\Omega)}, R, T\right)+\left\|u_{x}(x, 0)\right\|_{L^{2}(\Omega)} T^{1 / 2} .
$$

Finally we estimate $\int_{\Omega}\left(u_{x x}\right)^{2} d x$. Recall that the solution $u(x, t)$ is in $H^{3}(\Omega)$ and observe that the boundary condition on $u$ implies that $u_{t x}=0$ on $\partial \Omega$. As a result

$$
\begin{aligned}
\int_{\Omega} u_{x x t} u_{x x} d x= & -\int_{\Omega} u_{x t} u_{x x x} d x \\
= & \int_{\Omega}-\frac{\left|u_{x x x}\right|^{2}}{1+w} d x+\left(\frac{u_{x x} w_{x}}{(1+w)^{2}}+\left[(1+w) u^{3}\right]_{x}-\left[\frac{w u}{1+w}\right]_{x}\right) u_{x x x} d x \\
\leq & \int_{\Omega} \frac{1}{4 \varepsilon_{1}} \frac{\left|w_{x}\right|^{2}\left|u_{x x}\right|^{2}}{(1+w)^{3}}+\frac{1}{4 \varepsilon_{2}}(1+w)\left((1+w) u^{3}\right)_{x}^{2} d x \\
& +\int_{\Omega} \frac{1}{4 \varepsilon_{3}}(1+w)\left[\frac{w u}{1+w}\right]_{x}^{2} d x,
\end{aligned}
$$

where $\varepsilon_{1}+\varepsilon_{2}+\varepsilon_{3} \leq 1$. According to the boundary data (see [7, Lemma A.1, p. 12])

$$
\int_{\Omega}\left|u_{x}(x, t)\right|^{2} \leq \nu_{1} \int_{\Omega}\left|u_{x x}(x, t)\right|^{2},
$$

where $\nu_{1}$ denotes the first positive eigenvalue of the Laplacian with homogeneous Neumann boundary data, we can see that

$$
\begin{aligned}
\int_{\Omega} u_{x x t} u_{x x} d x \leq & C_{1}\left(\left\|w_{0}\right\|_{H^{2}(\Omega)},\left\|u_{0}\right\|_{L^{\infty}(\Omega)}, R, T, \varepsilon_{1}, \varepsilon_{2}, \varepsilon_{3}, \nu_{1}\right) \int_{\Omega}\left|u_{x x}(x, t)\right|^{2} \\
& +C_{2}\left(\left\|w_{0}\right\|_{H^{2}(\Omega)},\left\|u_{0}\right\|_{L^{\infty}(\Omega)}, R, T, \varepsilon_{1}, \varepsilon_{2}, \varepsilon_{3}\right) .
\end{aligned}
$$


Thus

$$
\int_{\Omega} u_{x x}^{2}(x, t) d x \leq\left(\left\|u_{x x}(x, 0)\right\|_{L^{2}(\Omega)}^{2}+\frac{C_{2}}{C_{1}}\right) e^{2 C_{1} t}-\frac{C_{2}}{C_{1}},
$$

where $C_{1}=C_{1}\left(\left\|w_{0}\right\|_{H^{2}(\Omega)},\left\|u_{0}\right\|_{L^{\infty}(\Omega)}, R, T, \varepsilon_{1}, \varepsilon_{2}, \varepsilon_{3}, \nu_{1}\right)$ and

$C_{2}=C_{2}\left(\left\|w_{0}\right\|_{H^{2}(\Omega)},\left\|u_{0}\right\|_{L^{\infty}(\Omega)}, R, T, \varepsilon_{1}, \varepsilon_{2}, \varepsilon_{3}\right)$. This gives us

$$
\left\|u_{x x}\right\|_{L^{2}\left(Q_{T}\right)} \leq\left[\left(\left\|u_{x x}(x, 0)\right\|_{L^{2}(\Omega)}^{2}+\frac{C_{2}}{C_{1}}\right) e^{2 C_{1} T}-\frac{C_{2}}{C_{1}}\right]^{1 / 2} T^{1 / 2} .
$$

As a consequence of the above we have that

$$
\left\|u_{t}\right\|_{L^{2}\left(Q_{T}\right)} \leq C\left(\left\|w_{0}\right\|_{H^{2}(\Omega)},\left\|u_{0}\right\|_{H^{2}(\Omega)}, R, T\right) T^{1 / 2},
$$

so we can conclude that for sufficiently small $\mathrm{T}$ and appropriate initial data $u(x, t) \in B$. This implies that $A$ maps $B$ for sufficiently small $T$ into $B$. Recalling (5.8) and (5.10) we see that the left hand side of (5.7) can be bounded by a constant that only depends on $R, T,\left\|u_{0}\right\|_{L^{\infty}(\Omega}$ and $\left\|w_{0}\right\|_{H^{2}(\Omega)}$. Thus $A$ in fact maps

$$
\begin{aligned}
B_{0}:= & \left\{f \in L^{2}\left(H^{3}(\Omega) ;(0, T)\right) ;\|f\|_{L^{2}\left(H^{2}(\Omega) ;(0, T)\right)} \leq R,\left\|f_{x}\right\|_{L^{2}\left(H^{2}(\Omega) ;(0, T)\right)} \leq C_{1},\right. \\
& \left.\left\|f_{t}\right\|_{L^{2}\left(H^{1}(\Omega) ;(0, T)\right)} \leq C_{2}, f(x, 0)=u_{0}(x) \text { in } \Omega\right\} \subset B
\end{aligned}
$$

(where $C_{1}$ and $C_{2}$ only depend on $\left\|u_{0}\right\|_{H^{2}(\Omega)},\left\|w_{0}\right\|_{H^{2}(\Omega)}, R$ and $T$ ) into itself. To apply Schauder's fixed point theorem we also have to show that $A$ is a weakly continuous mapping from $B_{0}$ to $B_{0}$, and for this we need an $H^{2}$-bound for the solution $v(x, t)$ of problem (5.6). We are therefore trying to find a $L^{2}$-bound of $v_{t}$. Differentiation of the equation (5.6) with respect to $t$ yields

$$
v_{t t}=a_{1}(w) v_{x x t}+a_{2}\left(w, w_{x}\right) v_{x t}+a_{3}(u, w) v_{t}+b\left(u, u_{t}, w, w_{x}, w_{t x}, v, v_{x}\right)
$$

with

$b\left(u, u_{t}, w, w_{x}, w_{t x}, v, v_{x}\right)=v_{x}\left(\frac{d}{d t} a_{2}\left(w, w_{x}\right)\right)+v\left(\frac{d}{d t} a_{3}(u, w)\right)+\frac{d}{d t} a_{4}\left(u, w, w_{x}\right)$.

Regarding our previous calculations and using the equation for $w(x, t)$, one can check that $b\left(u, u_{t}, w, w_{x}, w_{t x}, v, v_{x}\right) \in L^{2}(\Omega)$ and that its $L^{2}(\Omega)$-norm can be bounded for all $t \in(0, T)$ by a constant depending only on $R, T$ and the $H^{2}$ norms of the initial data $u_{0}$ and $w_{0}$. Testing equation (5.18) with $v_{t}$ we can also bound the $L^{2}$-norm of $v_{t}$ for all $t \in(0, T)$ by a constant depending only on these parameters. However this implies that after testing the equation (5.6) with $v_{t}$, we find a bound on $\left\|v_{x x}\right\|_{L^{2}(\Omega)}\left(=\left\|u_{x x x}\right\|_{L^{2}(\Omega)}\right)$ for all $t \in(0, T)$ that depends only on $R, T$ and the $H^{2}$-norms of the initial data $u_{0}$ and $w_{0}$.

This and the previous estimates for the functions $u(x, t)$ and $u_{x}(x, t)$ together with Sobolev's embedding theorems for $N=1$ guarantee the weak continuity of the 
mapping $A$ from $B_{0}$ to $B_{0}$. Thus, taking a sequence $\left(f_{i}\right)_{i \in \mathbb{N}}$ in $B_{0}$ we see that this sequence contains a weakly convergent (in $L^{2}\left((0, T) ; H^{3}(\Omega)\right)$ ) subsequence once again denoted with $\left(f_{i}\right)_{i \in \mathbb{N}}$ which converges to some function $f$ in $B_{0}$. The corresponding $u_{i}:=A f_{i}$ converge weakly to some function $u \in B_{0}$. The estimates above allow us to pass to the limit and we get that $u:=A f$. According to Schauder's fixed point theorem there exists a fixed point $u \in B$ with $u=A u$. This completes the local existence proof.

The regularity of the solution follows directly from the regularity of the solution of problem (5.5).

Using this we obtain the local existence of a classical solution of equation (5.1), as summarized in the following.

Corollary 5.2 Suppose $p_{0}(x)$ and $w_{0}(x)$ are positive functions from the class $C^{2, \beta}(\Omega)(\beta \in(0,1))$ satisfying the compatibility condition $\left(\frac{p_{0}}{1+w_{0}}\right)_{x}=0$ on $\partial \Omega$. Then there is a time $T>0$ such that there exists a solution $(p(x, t), w(x, t))$ of (5.1) with

$$
p \in L^{\infty}\left(L_{+}^{\infty}(\Omega) ;(0, T)\right) \cap L^{2}\left(H^{2}(\Omega) ;(0, T)\right), p_{t} \in L^{2}\left(Q_{T}\right)
$$

and $w \in L^{\infty}\left(L_{+}^{\infty}(\Omega) ;(0, T)\right) \cap L^{2}\left(H^{2}(\Omega) ;(0, T)\right), w_{t} \in L^{2}\left(H^{2}(\Omega) ;(0, T)\right)$.

Furthermore this solution is unique with $p(x, t) \geq 0$ and $w(x, t) \geq 0$ for all $t \in$ $(0, T)$. Finally $p(x, t) \in C^{1+\frac{\beta}{2}, 2+\beta}\left(Q_{T}\right)$ and consequently $w(x, t) \in C^{2+\frac{\beta}{2}, 2+\beta}\left(Q_{T}\right)$.

Remark 5.3 To obtain the existence result for the system (5.1) we must increase the regularity on $w_{0}(x)$ to guarantee that the corresponding function $u_{0}:=\frac{p_{0}}{1+w_{0}}$ belongs to $C^{2, \beta}(\Omega)$. One might be able to obtain better regularity results for the solution, but that is not the goal of the present paper. The uniqueness statement mentioned in Corollary 5.2 can be found in detail in the Appendix of the present paper.

The same arguments also work to prove the local existence of a solution of the problem

$$
\left.\begin{array}{rlrl}
p_{t} & =\left(\frac{p}{1+w^{2}}+\alpha p\right)_{x x}, & & \text { in } Q_{T} \\
\epsilon w_{t} & =p-\mu w, & & \text { in } Q_{T} \\
\left.\frac{p}{1+w^{2}}\right)_{x} & =0, & & \text { on } \Gamma_{T} \\
p(x, 0) & =p_{0}(x), w(x, 0)=w_{0}(x), & & \text { in } \Omega .
\end{array}\right\}
$$

Here we have to introduce the new function

$$
u(x, t):=\frac{p}{1+w^{2}}+\alpha p
$$


and are thus led to the problem

$$
\left.\begin{array}{rlrl}
u_{t} & =\left(\frac{1}{1+w^{2}}+\alpha\right) u_{x x}-\frac{2 w\left(1+w^{2}\right) u^{2}-2 \mu w^{2} u}{\left(1+w^{2}\right)\left(1+\alpha+\alpha w^{2}\right)}, & & \text { in } Q_{T} \\
\epsilon w_{t} & =\frac{(1+w) u}{1+\alpha+\alpha w^{2}}-\mu w, & & \text { in } Q_{T} \\
u_{x} & =0, & & \text { on } \Gamma_{T} \\
p(x, 0) & =p_{0}(x), w(x, 0)=w_{0}(x), & & \text { in } \Omega .
\end{array}\right\}
$$

We also have an energy functional for (5.1), as for problem $(3.1)^{4}$ We define

$$
\mathcal{E}(t):=\int_{\Omega} \frac{p^{2}(x, t)+1}{1+w(x, t)}+\log (1+w(x, t)) d x .
$$

Since $\mathcal{E}(t)$ is bounded from below, we see that solutions of (3.1) either converge to a stationary solution as $t \rightarrow \infty$ or blow up in finite time.

Figure 5 shows the influence of the time delay that arises from incorporation of a finite reaction rate of the control substance on the blow-up behavior of the solution. Figure 5 (left) demonstrates that the finite rate provides an alternative form of regularization of the solution, in that the high frequency oscillations that exist in the singular solution shown in Figure 2 are absent. A similar comparison holds for Figure 5 (right) and Figure 3, and both cases show that the blowup is not solely dependent on the ill-posed character of the singular problem. However the characterization of the initial data that leads to blow-up is more complicated than in the singular model, in that the shape of the initial control substance distribution may control blowup. This is clarified in the following theorem, which is the analog for problem (5.1) of Theorem 3.6.
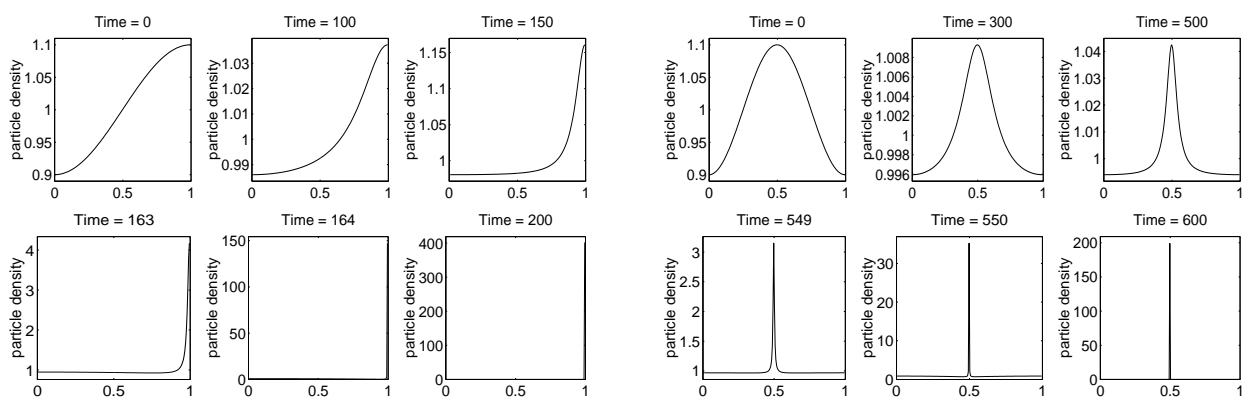

Figure 5. Smooth blowup of the solution of (5.1) for initial data $p(x, 0)=10-$ $0.1 \cos (\pi x)$ and $w(x, 0)=100.0$ (on the left) and $p(x, 0)=10-0.1 \cos (2 \pi x)$ and $w(x, 0)=100.0$ (on the right). In both simulations $\epsilon=1$.

${ }^{4}$ This is an important difference between system (5.1) and (5.19): for the latter there seems to be no Lyapunov function. 
Theorem 5.4 Assume that

$$
\int_{\Omega} \frac{p_{0}^{2}(x)+1}{1+w_{0}(x)}+\log \left(1+w_{0}(x)\right) d x<|\Omega|+\int_{\Omega} \log \left(1+M^{2}\right) d x
$$

where $\int_{\Omega} p_{0}(x) d x=M|\Omega|$. Then the solution of (5.1) must blow up in finite time, i.e. there exists a maximal time $T_{\max }<\infty$ such that $\|p(x, t)\|_{H^{2}(\Omega)} \rightarrow \infty$ as $t \rightarrow T_{\max }$.

Proof of Theorem 5.4: We can argue in the same way as for Theorem 3.6. We see that

$$
\frac{d}{d t} \mathcal{E}(t)=\int_{\Omega} \frac{2 p_{t} p+w_{t}}{1+w}-\frac{w_{t}\left(p^{2}+1\right)}{(1+w)^{2}}=-\int_{\Omega} 2\left|\left(\frac{p}{1+w}\right)_{x}\right|^{2}+\frac{\epsilon w_{t}^{2}}{(1+w)^{2}} d x
$$

If we assume that $T_{\max }=\infty$ we can conclude that

$$
\lim _{t \rightarrow \infty} 2 \int_{\Omega}\left|\left(\frac{p}{1+w}\right)_{x}\right|^{2} d x=0 \text { and } \lim _{t \rightarrow \infty} \int_{\Omega} \frac{\epsilon w_{t}^{2}}{(1+w)^{2}} d x=0
$$

since $\mathcal{E}(t)$ is bounded from below by 0 . This implies that the solution must converge to a stationary solution of (5.1) as $t \rightarrow \infty$. However, the only continuous stationary solution satisfying the mass constraint is the constant solution $p(x, t)=M$, $w(x, t)=M^{2}$. But this is not possible from our assumptions on the initial data. Thus the solution has to blow up in finite time.

Remark 5.5 We proved the local existence of a solution belonging to $H^{2}(\Omega)$. Even if it is possible to prove the local existence of a solution belonging only to $H^{1}(\Omega)$ under weaker assumptions than those we assumed in Corollary 5.2, the finite time blow-up result remains valid by replacing the $H^{2}$-norm by the $H^{1}$ norm. Once again we see from the numerical calculations that the $L^{\infty}$-norm is the correct blow-up norm in this case as well, but we have no analytical proof for this observation.

In contrast to problem (3.1), we are not able to characterize a set of initial data for which the solution exists globally in time. Numerical experiments show that this depends on the shape of the initial data $w(x, 0)$, but we do not know what conditions, other than the energy condition, classify the initial data for (5.1) according to whether the solutions exist for all time or blow up in finite time. Moreover, in the latter case the number of blowup points depends on $\epsilon$ as well. Figure 6 shows how the magnitude of $\epsilon$ affects the number of possible blow-up points, all other factors being equal. 
Finally, observe that model (3.1) and model (5.1) have the same steady state solutions, which is in contrast to the Cahn-Hilliard regularization, where the steady state does not have to be a constant solution. From the perspective of our studies of the least energy solution, the regularized model is more closely related to the singular equation (3.1) than the "Cahn-Hilliard" approach.
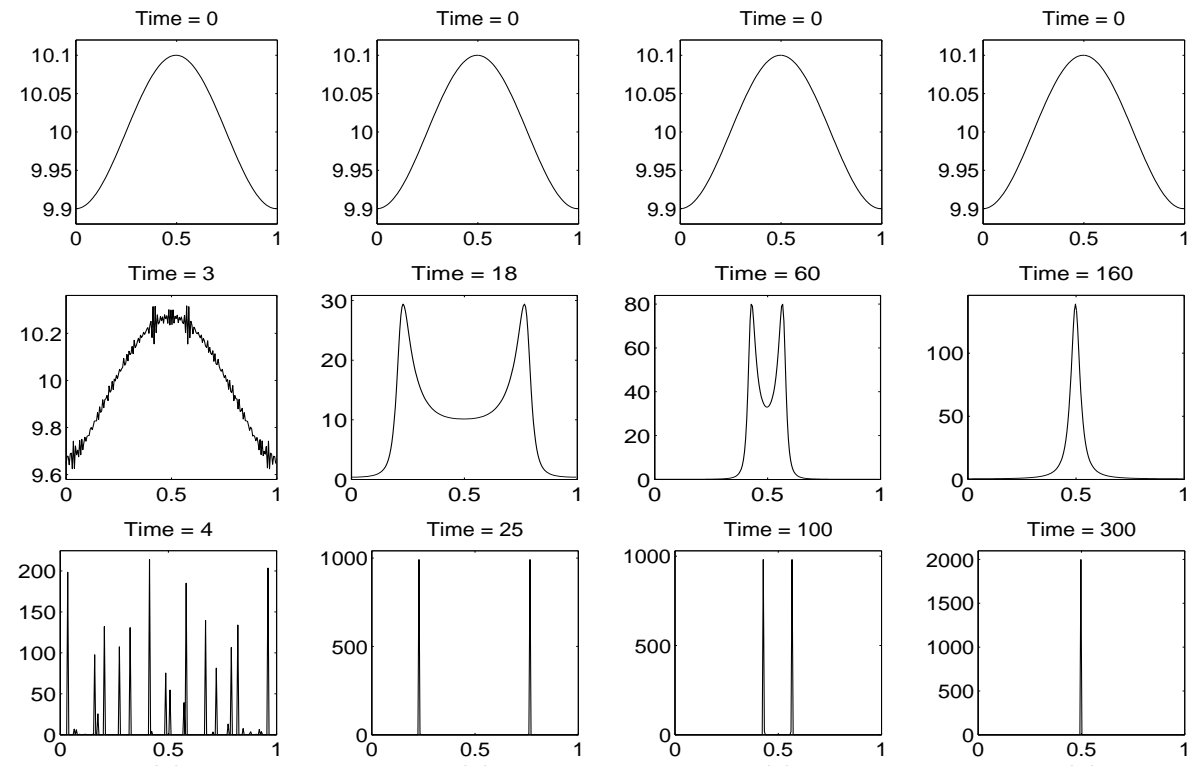

(a)

(b)

(c)

(d)

Figure 6. Multiple peak blow-up for different values of $\epsilon$ : (a) 0.1 (b) 1.0 (c) 5.0 and

(d) 25. Initial data is given by $p(x, 0)=10-0.1 \cos (2 \pi x)$ and $w(x, 0)=100$.

Remark 5.6 The incorporation of a small random diffusion component into the probability of jump can be modeling by considering $\mathcal{T}(p)$ of the form $\alpha p+1 /(1+$ $p^{2}$ ) either for the singular model (3.1) or model (5.1). Simulations (see [22]) for sufficiently large $\alpha$ demonstrate evolution to the homogeneous solution even for supercritical initial data. At lower values of $\alpha$, the early evolution is similar to the results for the singular system described in Section 4, with multiple peak blowup followed by coarsening. However, the long term evolution is different, with evolution to stable multiple peak solutions rather than a single peak.

\section{Conclusion and open questions}

We have studied a continuum formulation of a reinforced random walk that may describe the motion of certain biological organisms. The analysis of the discrete 
(lattice-based) version of the problem has been carried out in [22]. Here we proved the global existence of the solution in the case of subcritical initial data and studied the time asymptotic behavior of the solution for general initial data. Our numerical experiments, which will be carried out in more detail in [23], showed that the solution of the forward-backward equation undergoes a coarsening effect for some supercritical initial data. We gave an intuitive explanation for the observed coarsening effect for the continuum problem that parallels what can be proven rigorously for the discrete problem analyzed in [22]. Using variational techniques, we gave some arguments as to why the final blow-up profile of solution is expected to be a single peak $\delta$-distribution (as has been observed in our studies of the discrete models in [22]).

In addition, we studied reaction-diffusion systems that arise from models of movement of mobile populations, and which can be interpreted as reasonable regularizations of the forward-backward equation and that display similar time asymptotics as for the singular problem. We established the well-posedness of these systems and studied the time asymptotic behavior of the solutions for different initial data. We also suggested possible criteria to classify the initial data leading to the convergence to the uniform distribution and those leading to blowup in finite time. Further numerical results on these issues will be reported in [23]. Unfortunately, in gaining the local existence of a solution for all sufficiently smooth initial data for the regularizations, we lost the rigorous characterization of one class of initial data which leads to solutions that exist globally in time. The loss of a maximum principle for the first equation of (5.1) does not allow us to make an analogous statement for this model.

As we have already mentioned in previous remarks, we cannot prove that the $L^{\infty}$-norm of a solution blows up for blow-up solutions. This brings us to open questions which might be worth studying. As mentioned previously it would be of interest to find a proof which shows that in fact the $L^{\infty}$-norm of the function $p$ blows up in finite time for initial data satisfying the energy condition in both models (3.1) and (5.1). Moreover, it would be interesting to establish a rigorous analytical classification of initial data that leads to finite time blow-up of the solution of the problem (5.1). Once again, the energy condition in Theorem 5.4 should imply properties on the initial data and comments made through out the paper give some insights on this condition. The numerical studies provide some information, but a general classification remains missing.

Finally, the question remains as to whether existence and blowup are dependent on the dimension of the underlying space. For example, the existence proof of a solution of problem (5.1) resp. (5.19) requires crucial Sobolev inequalities, which are only valid in one dimension, and therefore it remains to be shown whether local existence holds in higher dimensions. Concerning blowup, computational results 
for the model studied in [21] indicate that axisymmetric blowup in two dimensions is essentially identical in both profile and blowup time to the one-dimensional case, but there are no rigorous results on this issue as yet.

\section{Acknowledgements}

Dirk Horstmann's research has been supported by the Deutsche Forschungsgemeinschaft (DFG). Kevin Painter's research has been supported by SHEFC grant 107. Hans Othmer's research is supported in part by NSF Grant DMS9805494 and NIH Grant GM29123.

\section{References}

[1] Adams, R.A. 1975 Sobolev Spaces. Academic Press. New York, San Francisco, London.

[2] Alikakos, N. $1979 L^{p}$ Bounds of solutions of reaction-diffusion equations. Comm. Partial Diff. Eqns. 4, 827-868.

[3] Alt, H.W. 1992 Lineare Funktionalanalysis. Springer Verlag. Berlin, Heidelberg, New York, 2. Auflage.

[4] Aronson, D. G. 1985 The role of diffusion in mathematical population biology: Skellam revisited. Mathematics in Biology and Medicine, editors $V$. Capasso and $E$. Grosso and S. L. Paveri-Fontana, 2-6.

[5] J. Billen, J \& Gobin, B. 1996 Trail following in army ants (Hymenoptera, Formicidae). Neth. J. Zool. 46, 272-280.

[6] Carr, J. \& Gurtin, M. E. \& Slemrod, M. 1984 Structured phase transitions on a finite interval. Arch. Rational Mech. Anal. 86, 317-351.

[7] Conway, E. \& Hoff, D. \& Smoller, J. 1978 Large time behavior of solutions of systems of nonlinear reaction-diffusion equations. SIAM J. Appl. Math. 35, 1-16.

[8] Elliott, C. M. 1989 The Cahn-Hilliard model for the kinetics of phase separation. Mathematical Models for Phase Change Problems, International Series of Numerical Mathematics, Vol. 88, Birkhäuser Verlag. Basel.

[9] Evans, L. C. 1998 Partial Differential Equations, American Mathematical Society, Graduate Studies in Mathematics 19, Providence, Rhode Island.

[10] B. Hölldobler and E. O. Wilson. Ants. Harvard University Press, Cambridge, 1990.

[11] Heuser, H. 1975 Funktionalanalysis, Teubner, Stuttgart.

[12] Kawohl, B. \& Kutev, N. 1998 Maximum principle and comparison theorems for anisotropic diffusion. Mathematische Annalen 311, 107-123.

[13] Kichenassamy, S. 1997 The Perona-Malik paradox. SIAM J. Appl. Math. 57, 13281342.

[14] Ladyzenskaya, O. A. \& Ural'ceva, N. N. 1968 Linear and quasilinear elliptic equations, Academic Press, New York, London.

[15] Ladyzenskaya, O. A. \& Solonnikov, V. A. \& Ural'ceva, N. N. 1969 Linear and quasilinear equations of parabolic type, Translations of Mathematical Monographs, Volume 23, AMS.

[16] F. Lutscher and A. Stevens 2001 Emerging patterns in a hyperbolic model for locally interacting cell systems. MPI-MIS Preprint no.: 98 (2001). 
[17] Levine, H. A. \& Sleeman, B. D. 1997 A system of reaction diffusion equations arising in the theory of reinforced random walks. SIAM J. Appled. Math. 57, 683-730.

[18] Othmer, H. G. 1969 Interactions of reaction and diffusion in open systems, Ph. D. Thesis, University of Minnesota.

[19] Othmer, H. G. \& Dunbar, S. R. \& Alt, W. Models of dispersal in biological systems. J. Math. Biol. 26, 263-298.

[20] Othmer, H. G. \& Schaap, P. 1998 Oscillatory cAMP Signaling in the Development of Dictyostelium discoideum, Comments on Theor. Biol. 5, 175-282.

[21] Othmer, H. G. \& Stevens, A. 1997 Aggregation, Blowup and Collapse: The ABC's of generalized taxis in reinforced random walks. SIAM J. Appled. Math. 57, 10441081.

[22] Painter, K. J. \& Horstmann, D. \& Othmer, H. G. 2002 Localization in Lattice and Continuum Models of Reinforced Random Walks, Appl. Math. Letters to appear.

[23] Painter, K. J. \& Horstmann, D. \& Othmer, H. G. Applications of models for localization of populations, in preparation.

[24] Patlak, C. S. 1953 Random walk with persistence and external bias. Bull. of Math. Biophys. 15, 311-338.

[25] Perona, P. \& Malik, J. 1990 Scale space and edge detection using anisotropic diffusion. IEEE Trans. Pattern Anal. Mach. Intell. 12, 629-639.

[26] Rosenau, P. 1987 Dynamics of dense lattice. Physical Review B 36, 5868-5876.

[27] Smoller, J. 1994 Shock waves and reaction-diffusion equations, Springer-Verlag, New York, Heidelberg, second edition.

[28] Struwe, M. 1996 Variational Methods, Springer Verlag Berlin, Heidelberg, New York, second edition.

\section{Appendix A Notation}

Throughout the paper we use the following notation. We write $u=\mathcal{O}(g)$ as $x \rightarrow x_{0}$, if there is a constant $C$ such that $|u(x)| \leq C|g(x)|$ holds for all $x$ which are sufficiently close to $x_{0} . \Omega \subset \mathbb{R}^{N}(N \geq 1)$ be an open set. We use the following notation for spaces of differentiable functions on $\Omega$.

- $C^{k}(\Omega):=\{u: \Omega \rightarrow \mathbb{R} \mid u$ is $k$-times continuous differentiable $\}$

- $C^{k}(\bar{\Omega}):\left\{u \in C^{k}(\Omega) \mid D^{\alpha} u\right.$ is continuous for all $\left.|\alpha| \leq k\right\}$,

- $C^{\infty}(\Omega):=\bigcap_{k=0}^{\infty} C^{k}(\Omega)$, and $C^{\infty}(\bar{\Omega}):=\bigcap_{k=0}^{\infty} C^{k}(\bar{\Omega})$.

For the Hölder continuous functions we use the notation

$$
C^{k, \beta}(\bar{\Omega}):=\left\{u \in C^{k}(\bar{\Omega}) \mid \sum_{|\alpha| \leq k}\left\|D^{\alpha} u\right\|_{C(\bar{\Omega})}+\sum_{|\alpha|=k}\left[D^{\alpha} u\right]_{C^{0, \beta}(\bar{\Omega})}<\infty\right\},
$$

where

$$
\|u\|_{C(\bar{\Omega})} \equiv \sup _{x \in \Omega}|u(x)|
$$


and

$$
[u]_{C^{0, \beta}(\bar{\Omega})} \equiv \sup _{x, y \in \Omega, x \neq y}\left\{\frac{|u(x)-u(y)|}{|x-y|^{\beta}}\right\} .
$$

We also use the following function spaces.

$$
L^{r}(\Omega):=\left\{u: \Omega \rightarrow \mathbb{R} \mid u \text { is measurable and }\|u\|_{L^{r}(\Omega)}<\infty\right\}
$$

where

$$
\begin{gathered}
\|u\|_{L^{r}(\Omega)}:=\left(\int_{\Omega}|u|^{r} d x\right)^{1 / r} \text { for } 1 \leq r<\infty . \\
L^{\infty}(\Omega):=\left\{u: \Omega \rightarrow \mathbb{R} \mid u \text { is measurable and }\|u\|_{L^{\infty}(\Omega)}<\infty\right\},
\end{gathered}
$$

where

$$
\|u\|_{L^{\infty}(\Omega)}:=\underset{\Omega}{\operatorname{ess} \sup }|u| .
$$

$$
\begin{aligned}
W^{k, r}(\Omega):= & \left\{u: \Omega \rightarrow \mathbb{R} \mid u \in L^{r}(\Omega) \text { and the weak derivatives } D^{\alpha} u\right. \text { exist } \\
& \text { for all multiindices } \left.\alpha \text { with }|\alpha| \leq k \text { and belong to } L^{r}(\Omega)\right\},
\end{aligned}
$$

where the norm in $W^{k, r}(\Omega)$ is given by

$$
\|u\|_{W^{k, r}(\Omega)}:=\left\{\begin{array}{ll}
\left(\sum_{|\alpha| \leq k} \int_{\Omega}\left|D^{\alpha} u\right|^{r} d x\right)^{1 / r}, & 1 \leq r<\infty \\
\sum_{|\alpha| \leq k}^{\operatorname{ess} \sup \left|D^{\alpha} u\right|,} & r=\infty
\end{array} .\right.
$$

We use the notation $H^{1}(\Omega)$ for functions belonging to $W^{1,2}(\Omega)$ and $H^{2}(\Omega)$ for functions belonging to $W^{2,2}(\Omega)$. We denote the dual space of $H^{1}(\Omega)$ by $\left(H^{1}(\Omega)\right)^{*}$.

Finally, the following notations are used for time dependent $L^{r}$-spacesi

$$
L^{r}(\mathcal{X} ;(0, T)):=\left\{u:[0, T] \rightarrow \mathcal{X} \mid u \text { is measurable and }\|u\|_{L^{r}(\mathcal{X} ;(0, T))}<\infty\right\},
$$

where

$$
\|u\|_{L^{r}(\mathcal{X} ;(0, T))}:=\left(\int_{0}^{T}\|u(t)\|_{\mathcal{X}}^{r} d t\right)^{1 / r}
$$

for $1 \leq r<\infty$ and,

for $r=\infty$.

$$
\|u\|_{L^{\infty}(\mathcal{X} ;(0, T))}:=\operatorname{ess}_{0 \leq t \leq T} \sup _{0 \leq u} \| \mathcal{X}
$$

$$
C(\mathcal{X} ;[0, T]):=\left\{u:[0, T] \rightarrow \mathcal{X} \mid u \text { is continuous and }\|u\|_{C(\mathcal{X} ;[0, T])}<\infty\right\},
$$


where

$$
\|u\|_{C(\mathcal{X} ;[0, T])}:=\max _{0 \leq t \leq T}\|u(t)\|_{\mathcal{X}}
$$

We write $L^{r}\left(\Omega_{T}\right)$ for the function spaces $L^{r}\left(L^{r}(\Omega) ;(0, T)\right)$ for all $1 \leq r \leq \infty$.

\section{Appendix B Proof of Corollary 5.2:}

Let us assume without loss of generality that $K=1$. As mentioned during the text, we see that the existence of a solution is a direct consequence of the existence of a solution for (5.2). Thus we only have to prove the uniqueness of this solution. So let us assume that there are two solutions $(p(x, t), w(x, t))$ and $(q(x, t), v(x, t))$ of $(5.1)$ with $p(x, 0)=p_{0}(x)=q(x, 0)$ and $w(x, 0)=w_{0}(x)=v(x, 0)$. We see that the following equalities hold:

$$
\begin{gathered}
\int_{\Omega}\left(p_{t}-q_{t}\right)(p-q) d x=\int_{\Omega}\left(\frac{p}{1+w}-\frac{p}{1+v}+\frac{p}{1+v}-\frac{q}{1+v}\right)_{x x}(p-q) d x \\
=\int_{\Omega}-\frac{\left|(p-q)_{x}\right|^{2}}{1+v}+\frac{p-q}{(1+v)^{2}} v_{x}(p-q)_{x} d x \\
\quad+\int_{\Omega}-\frac{v-w}{(1+w)(1+v)} p_{x}(p-q)_{x} d x \\
\quad+\int_{\Omega} p(p-q)_{x}\left(\frac{w_{x}}{(1+w)^{2}}-\frac{v_{x}}{(1+v)^{2}}\right) d x \\
=I_{1}+I_{2}+I_{3}+I_{4} \\
\int_{\Omega}\left(w_{t}-v_{t}\right)(w-v) d x=\int_{\Omega}(p+q)(p-q)(w-v)-(w-v)^{2} d x \\
=I I_{1}+I I_{2}
\end{gathered}
$$

and

$$
\begin{aligned}
\int_{\Omega}\left(w_{x t}-v_{x t}\right)\left(w_{x}-v_{x}\right) d x= & \int_{\Omega}(p+q)(p-q)_{x}(w-v)_{x} d x \\
& +\int_{\Omega}(p-q)(p+q)_{x}(w-v)_{x}-\left|(w-v)_{x}\right|^{2} d x \\
= & I I I_{1}+I I I_{2}+I I I_{3} .
\end{aligned}
$$

Let us now add these three identities and we get the equality

$$
\frac{d}{d t} J(t)=\sum_{i=1}^{4} I_{i}+\sum_{i=1}^{2} I I_{i}+\sum_{i=1}^{3} I I I_{i},
$$


where $J(t):=\|p-q\|_{L^{2}(\Omega)}^{2}+\|w-v\|_{L^{2}(\Omega)}^{2}+\left\|(w-v)_{x}\right\|_{L^{2}(\Omega)}^{2}$. Before we proceed further on we remark that we can estimate $I_{4}$ from above by

$$
\begin{aligned}
I_{4} \leq & \left\|p(1+v)^{1 / 2}\right\|_{L^{\infty}(\Omega)}\left\|\frac{(p-q)_{x}}{(1+v)^{1 / 2}}\right\|_{L^{2}(\Omega)}\left\|\frac{w_{x}}{(1+w)^{2}}-\frac{v_{x}}{(1+v)^{2}}\right\|_{L^{2}(\Omega)} \\
\leq & \left\|p(1+v)^{1 / 2}\right\|_{L^{\infty}(\Omega)}\left\|\frac{(p-q)_{x}}{(1+v)^{1 / 2}}\right\|_{L^{2}(\Omega)} \\
& \times\left(\left.\left\|\frac{(w+v)(w-v)}{(1+w)^{2}(1+v)^{2}} w_{x}\right\|\right|_{L^{2}(\Omega)}+\left\|(w-v)_{x}\right\|_{L^{2}(\Omega)}\right) \\
\leq & \left\|p(1+v)^{1 / 2}\right\|_{L^{\infty}(\Omega)}\left\|\frac{(p-q)_{x}}{(1+v)^{1 / 2}}\right\|_{L^{2}(\Omega)}\|w-v\|_{L^{2}(\Omega)} \\
& \times\left.\left\|\frac{(w+v)}{(1+w)^{2}(1+v)^{2}} w_{x}\right\|\right|_{L^{\infty}(\Omega)}\left\|\frac{(p-q)_{x}}{(1+v)^{1 / 2}}\right\|\left\|_{L^{2}(\Omega)}\right\|(w-v)_{x} \|_{L^{2}(\Omega)}
\end{aligned}
$$

using Hölder's inequality. By using Hölder's inequality also on $I_{2}, I_{3}, I I_{1}, I I I_{1}$ and $I I I_{2}$ and then applying Cauchy's inequality on each of these terms we derive 
the inequality

$$
\begin{aligned}
\frac{d}{d t} J(t) \leq & \frac{1}{4 \varepsilon_{1}}\left\|\frac{\left|w_{x}\right|^{2}}{(1+v)^{3}}\right\|_{L^{\infty}(\Omega)}\|p-q\|_{L^{2}(\Omega)}^{2} \\
& +\left.\frac{1}{4 \varepsilon_{2}}\left\|\frac{\left|p_{x}\right|^{2}}{(1+v)(1+w)^{2}}\right\|\right|_{L^{\infty}(\Omega)}\|w-v\|_{L^{2}(\Omega)}^{2} \\
& +\frac{1}{4 \varepsilon_{3}}\left\|\frac{\left|p_{x}\right|^{2}}{(1+v)(1+w)^{2}}\right\|_{L^{\infty}(\Omega)}\|w-v\|_{L^{2}(\Omega)}^{2} \\
& +\frac{1}{4 \varepsilon_{4}}\left\|p(1+v)^{1 / 2}\right\|_{L^{\infty}(\Omega)}^{2}\left\|\frac{(w+v)\left|w_{x}\right|}{(1+w)^{2}(1+v)^{2}}\right\|_{L^{\infty}(\Omega)}^{2}\|w-v\|_{L^{2}(\Omega)}^{2} \\
& +\frac{1}{2}\left\|(p+q)^{2}\right\|_{L^{\infty}(\Omega)}\|p-q\|_{L^{2}(\Omega)}^{2}+\frac{3}{2}\|w-v\|_{L^{2}(\Omega)}^{2} \\
& +\frac{1}{2}\left\|\left(\left|(p+q)_{x}\right|\right)^{2}\right\| L_{L^{\infty}(\Omega)}\left\|(w-v)_{x}\right\|_{L^{2}(\Omega)}^{2} \\
& +\frac{1}{2}\|p-q\|_{L^{2}(\Omega)}^{2}+\frac{1}{4 \varepsilon_{5}}\left\|(1+v)(p+q)^{2}\right\|_{L^{\infty}(\Omega)}\left\|(w-v)_{x}\right\|_{L^{2}(\Omega)}^{2} \\
& -\left\|(w-v)_{x}\right\|_{L^{2}(\Omega)}^{2}+\frac{1}{4 \varepsilon_{6}}\left\|p(1+v)^{1 / 2}\right\|_{L^{\infty}(\Omega)}^{2}\left\|(w-v)_{x}\right\|_{L^{2}(\Omega)}^{2} \\
\leq & \frac{1}{4 \varepsilon_{1}}\left\|\left(\left|w_{x}\right|^{2}\right)\right\|_{L^{\infty}(\Omega)}\|p-q\|_{L^{2}(\Omega)}^{2}+\frac{1}{4 \varepsilon_{2}}\left\|\left(\left|p_{x}\right|^{2}\right)\right\|_{L^{\infty}(\Omega)}\|w-v\|_{L^{2}(\Omega)}^{2} \\
& +\frac{1}{4 \varepsilon_{4}}\left\|p(1+v)^{1 / 2}\right\|\left\|_{L^{\infty}(\Omega)}^{2}\right\|\left((w+v)\left|w_{x}\right|\right)\left\|_{L^{\infty}(\Omega)}^{2}\right\| w-v \|_{L^{2}(\Omega)}^{2} \\
& +\frac{1}{4 \varepsilon_{3}}\left\|\left(\left|p_{x}\right|\right)^{2}\right\|_{L^{\infty}(\Omega)}\|w-v\|_{L^{2}(\Omega)}^{2}+\frac{1}{2}\left\|(p+q)^{2}\right\|\left\|_{L^{\infty}(\Omega)}\right\| p-q \|_{L^{2}(\Omega)}^{2} \\
& +\frac{3}{2}\|w-v\|_{L^{2}(\Omega)}^{2}+\frac{1}{2}\left\|\left(\left|(p+q)_{x}\right|\right)^{2}\right\|_{L^{\infty}(\Omega)}\left\|(w-v)_{x}\right\|_{L^{2}(\Omega)}^{2} \\
& +\frac{1}{2}\|p-q\|_{L^{2}(\Omega)}^{2}+\frac{1}{4 \varepsilon_{5}}\left\|(1+v)(p+q)^{2}\right\|_{L^{\infty}(\Omega)}\left\|(w-v)_{x}\right\|_{L^{2}(\Omega)}^{2} \\
& -\left\|(w-v)_{x}\right\|_{L^{2}(\Omega)}^{2}+\frac{1}{4 \varepsilon_{6}}\left\|p(1+v)^{1 / 2}\right\|_{L^{\infty}(\Omega)}^{2}\left\|(w-v)_{x}\right\|_{L^{2}(\Omega)}^{2} \\
\leq & c o n s t\left(\|p-q\|_{L^{2}(\Omega)}^{2}+\|w-v\|_{L^{2}(\Omega)}^{2}+\left\|(w-v)_{x}\right\|_{L^{2}(\Omega)}^{2}\right) \\
= & \operatorname{lonst} \cdot J(t)
\end{aligned}
$$

with $\sum_{i=1}^{6} \varepsilon_{i}=1$. Gronwall's Lemma now implies the uniqueness of the solution. If we interpret the first equation of (5.1) as a linear parabolic equation with discontinuous coefficients, we see that all the coefficients satisfy the conditions of Theorem 12.1 Chapter III in [15] and we get the regularity for $p$ from this theorem. The 
regularity for $w$ is a direct consequence from the representation formula

$$
w(x, t)=w_{0}(x) e^{-t}+\int_{0}^{t} e^{s-t} p^{2}(s, x) d s .
$$

This completes the proof of the corollary. 